\title{
Article \\ MiR-106b-5p: A Master Regulator of Potential Biomarkers for
Breast Cancer Aggressiveness and Prognosis
}

\author{
Paula Lucía Farré ${ }^{1}$,, Rocío Belén Duca ${ }^{1,+}$, Cintia Massillo ${ }^{1,+}{ }^{\dagger}$, Guillermo Nicolás Dalton ${ }^{1}$, Karen Daniela Graña ${ }^{1}$, \\ Kevin Gardner ${ }^{2}$, Ezequiel Lacunza ${ }^{3}$ and Adriana De Siervi ${ }^{1, *}$ \\ 1 Laboratorio de Oncología Molecular y Nuevos Blancos Terapéuticos, Instituto de Biología y Medicina \\ Experimental (IBYME), CONICET, Buenos Aires C1428ADN, Argentina; pfarre@dna.uba.ar (P.L.F.); \\ rduca@dna.uba.ar (R.B.D.); cmassillo@dna.uba.ar (C.M.); ndalton@dna.uba.ar (G.N.D.); \\ kgrana@dna.uba.ar (K.D.G.) \\ 2 Department of Pathology and Cell Biology, Columbia University Medical Center, 630 W. 168th Street, \\ New York, NY 10032, USA; klg2160@cumc.columbia.edu \\ 3 Centro de Investigaciones Inmunológicas Básicas y Aplicadas (CINIBA), Facultad de Ciencias Médicas, \\ Universidad Nacional de La Plata, Buenos Aires B1900, Argentina; ezequiellacunza@hotmail.com \\ * Correspondence: adesiervi@dna.uba.ar; Tel.: +54-11-4783-2869 (ext. 1206) \\ + Both equally contributed to this work.
}

\section{check for} updates

Citation: Farré, P.L.; Duca, R.B.; Massillo, C.; Dalton, G.N.;

Graña, K.D.; Gardner, K.; Lacunza, E.; De Siervi, A. MiR-106b-5p: A Master Regulator of Potential Biomarkers for Breast Cancer Aggressiveness and Prognosis. Int. J. Mol. Sci. 2021, 22, 11135. https://doi.org/10.3390/ ijms222011135

Academic Editor:

Panayiotis A. Theodoropoulos

Received: 15 September 2021

Accepted: 12 October 2021

Published: 15 October 2021

Publisher's Note: MDPI stays neutral with regard to jurisdictional claims in published maps and institutional affiliations.

Copyright: (c) 2021 by the authors. Licensee MDPI, Basel, Switzerland. This article is an open access article distributed under the terms and conditions of the Creative Commons Attribution (CC BY) license (https:/ / creativecommons.org/licenses/by/ $4.0 /)$.

\begin{abstract}
Breast cancer (BCa) is the leading cause of death by cancer in women worldwide. This disease is mainly stratified in four subtypes according to the presence of specific receptors, which is important for BCa aggressiveness, progression and prognosis. MicroRNAs (miRNAs) are small noncoding RNAs that have the capability to modulate several genes. Our aim was to identify a miRNA signature deregulated in preclinical and clinical BCa models for potential biomarker discovery that would be useful for BCa diagnosis and/or prognosis. We identified hsa-miR-21-5p and miR-106b-5p as up-regulated and hsa-miR-205-5p and miR-143-3p as down-regulated in BCa compared to normal breast or normal adjacent (NAT) tissues. We established 51 shared target genes between hsa-miR21-5p and miR-106b-5p, which negatively correlated with the miRNA expression. Furthermore, we assessed the pathways in which these genes were involved and selected 12 that were associated with cancer and metabolism. Additionally, GAB1, GNG12, HBP1, MEF2A, PAFAH1B1, PPP1R3B, RPS6KA3 and SESN1 were downregulated in BCa compared to NAT. Interestingly, hsa-miR-106b-5p was up-regulated, while GAB1, GNG12, HBP1 and SESN1 were downregulated in aggressive subtypes. Finally, patients with high levels of hsa-miR-106b-5 and low levels of the abovementioned genes had worse relapse free survival and worse overall survival, except for GAB1.
\end{abstract}

Keywords: breast cancer; biomarker; aggressiveness; prognosis

\section{Introduction}

Breast cancer $(\mathrm{BCa})$ is the first in incidence and the leading cause of death by cancer among women worldwide [1]. It is a heterogeneous disease, since it has a variety of histological and biological properties in terms of genetics and epigenetics, which influences its diagnosis, treatment and prognosis. Even though there are plenty of treatments for this disease, the need of new therapeutic targets is urgent, since there is no specific treatment for the most aggressive BCa types.

Currently, $\mathrm{BCa}$ is stratified according to its staging and to its molecular subtype; this combination will determine the patient's therapy [2]. In terms of staging, it can be stratified from 0 to IV according to the size of the tumor (stages I-III), the presence of secondary tumors in nodes (stages II-III), their distance and the presence of metastasis in other organs (stage IV) [3]. According to its molecular profile, BCa is mostly divided according to the presence/absence of progesterone receptor (PR), estrogen receptor (ER) and/or ErbB2 receptor (Her2) [4]. 
The main subtypes are: Luminal $\mathrm{A}\left(\mathrm{PR}^{+}, \mathrm{ER}^{+}\right.$and Her2 $\left.{ }^{-}\right)$, Luminal $\mathrm{B}\left(\mathrm{PR}^{+}, \mathrm{ER}^{+}\right.$ and Her2 $\left.{ }^{+}\right)$, Her2 $\left(\mathrm{PR}^{-}, \mathrm{ER}^{-}\right.$and Her2 $\left.{ }^{+}\right)$and triple negative $\left(\mathrm{PR}^{-}, \mathrm{ER}^{-}\right.$and Her2 $\left.{ }^{-}\right)$, represented mostly by the basal-like sub-classification [5]. The luminal subtype is the most prevalent, with a better prognosis and several treatments available [4]. The Her2 subtype also has anti-receptor treatment, but has a worse prognosis compared to the luminal subtype. Finally, triple negative BCa has no specific treatment available thus far; therefore, it has the worse prognosis and is, therefore, the most aggressive subtype [4].

MicroRNAs (miRNAs) are small non-coding RNAs (18-22 nucleotides) capable of modulating gene-expression [6]. It was found that miRNAs play a crucial role in cancer development and progression including $\mathrm{BCa}[7,8]$ and can affect several hallmarks of cancer, such as cell death resistance, invasion and metastasis activation, among others [9]. In conclusion, miRNAs can play a role acting as oncomiRs or tumor suppressor miRNAs, mostly depending on their microenvironment [10].

The aim of this work was to identify a miRNA signature deregulated in breast tumors and their target genes as possible biomarkers for BCa diagnosis and/or prognosis. We compared the expression between normal breast/normal adjacent and BCa tissues of several miRNAs from mice model and human samples. Then, we focused on hsa-miR-21$5 p$ and miR-106b-5p that shared similar profiles between human and mice and identified their target genes. Using bioinformatics approaches, we evaluated the pathways in which these genes are involved, and their relevance in normal and cancerous tissues. We also established the overall survival and relapse free survival analysis in terms of miRNA and gene expression. Finally, the correlation between the expression of miRNAs and genes and $\mathrm{BCa}$ aggressiveness was assessed.

\section{Results}

\subsection{BCa Modulates miRNA Expression in $4 T 1$ Allografts and Human Tissue Samples}

In order to evaluate the expression of several miRNAs that have been reported as related to $\mathrm{BCa}$ development and progression [11-15], we developed a triple negative $\mathrm{BCa}$ (TNBC) mice model. Balb/c animals were randomly divided into two groups (BCa or control) and inoculated or not with $4 \mathrm{~T} 1$ cells. Four weeks after cell inoculation, all mice were sacrificed and tissues were collected. MiRNAs from mammary gland (MG) and tumors were isolated and assessed by stem-loop RT-qPCR (Figure 1A). We found that mmu-miR-21a-5p, miR-106b-5p, miR-125b-5p and miR-221-3p were upregulated ( $p$-values: $<0.0001,<0.0001,0.0011$ and 0.0027 , respectively) in $4 \mathrm{~T} 1$ allografts compared with normal MG; meanwhile mmu-miR-138-5p, miR-143-3p, miR-146a-5p and miR-205-5p were downregulated ( $p$-values: $<0.0001,<0.0001,<0.0001$ and 0.0002 respectively).

To analyze the expression profile of these miRNAs in BCa and normal adjacent tissue (NAT) from patients, available data was obtained from the UCSC Xena bioinformatic tool [16]. The information of the mature miRNAs expression obtained by RNAseq was analyzed using paired BCa-NAT from the same patient (Figure 1B). We found that hsa-125b$5 p$, miR-221-3p, miR-143-3p and miR-205-5p were downregulated in BCa tissue compared to NAT, while hsa-miR-21-5p and miR-106b-5p were upregulated. Since mice and human share several miRNA sequences and combining human and murine data, we found that miR-21-5p and miR-106b-5p were both up-regulated in mice and human tumors compared to MG or NAT, respectively, while miR-205-5p and miR-143-3p were both down-regulated. We next focused the analysis on these four miRNAs. 
A.
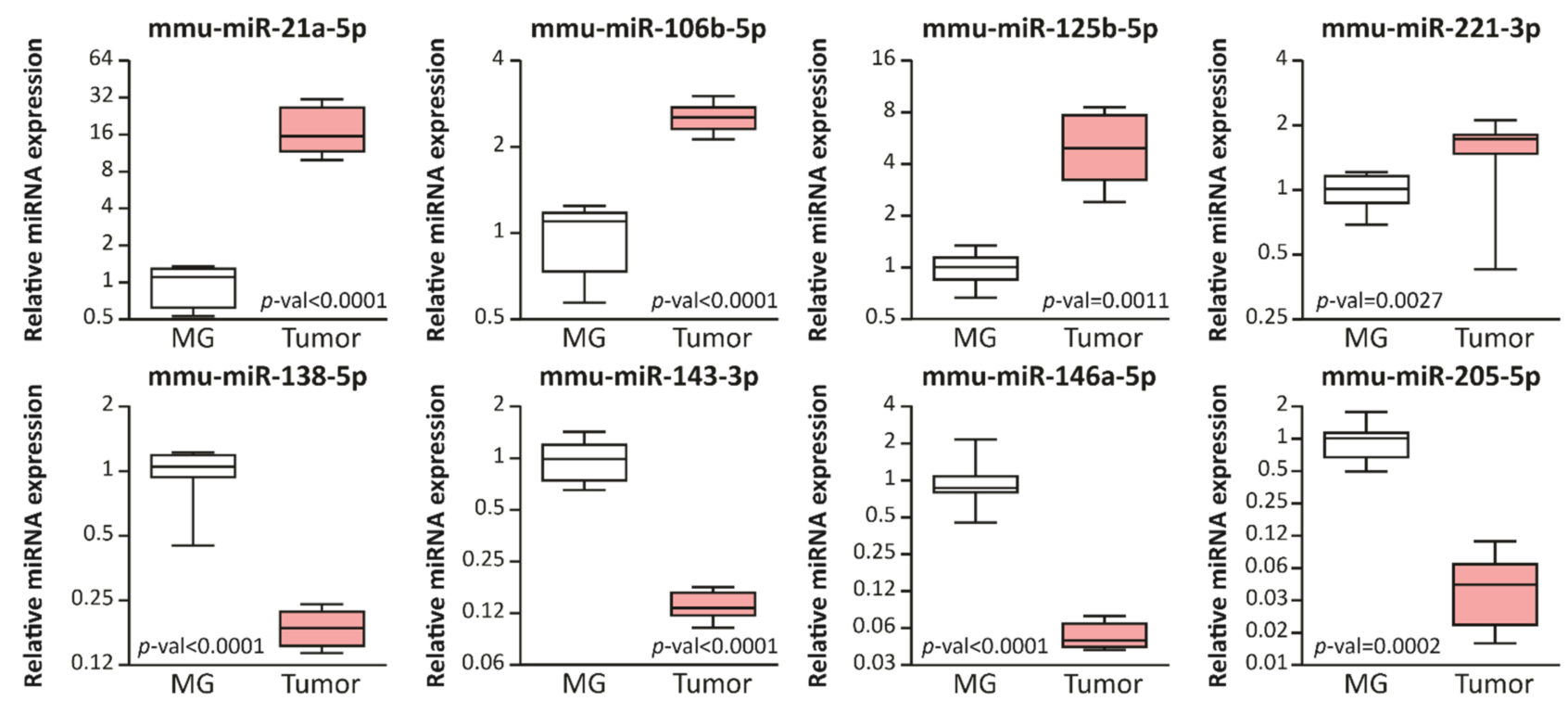

B.
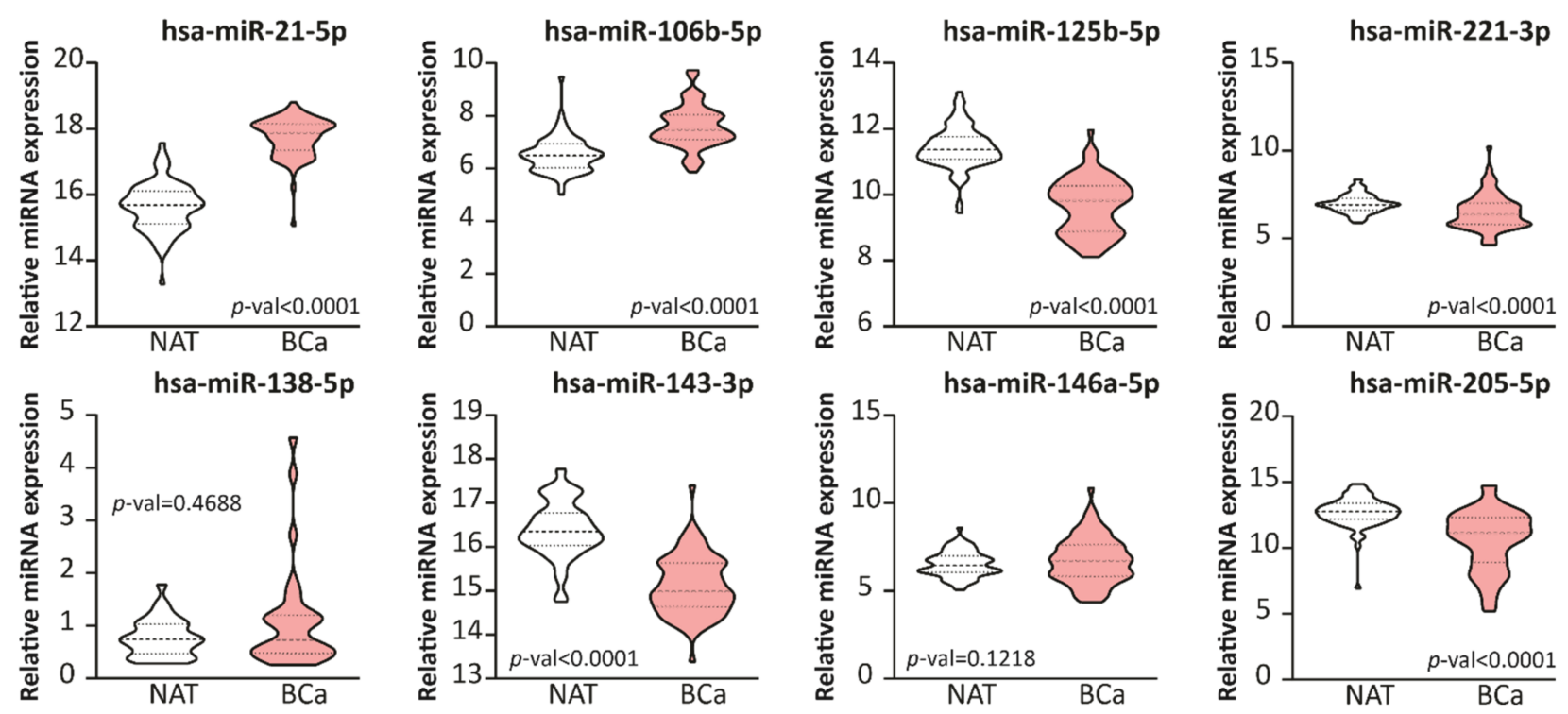

Figure 1. miRNAs expression is modulated in BCa mice and human samples. (A) Stem-loop RT-qPCR from 4T1 allografts (Tumor) or mammary-glands (MG) Balb/c mice using specific primers for the indicated miRNAs is shown $(n=8$ per group; primers were run in duplicates). The data were normalized to mmu-miR-191-5p and MG. Statistical analysis was performed using a T-test, Welch or sing-median test when appropriate. (B) Expression levels of the corresponding miRNAs in BCa tumors paired normal adjacent tissue (NAT) from the TCGA-BRCA dataset are graphed in read per millions. The name of the miRNAs is indicated up each plot in bold. Statistical analysis was performed using a paired T-test or Wilcoxon test when appropriate. 


\subsection{Hsa-miR-21-5p and miR-106b-5p Share Several Target Genes}

We obtained a list of experimentally validated target (EVT) genes for hsa-miR-21-5p, miR-106b-5p, miR-205-5p and miR-143-3p, using the DIANA TARBASE v8 resource. To identify common target genes, we used Venn diagrams (Figure 2A, Table S1). Based on this analysis, two miRNAs-target gene sets were obtained. The first one included the target genes modulated exclusively by the up-regulated miRNAs hsa-miR-21-5p and miR106b-5p, excluding those eight genes shared with hsa-miR-143-3p and miR-205-5p. The second one included the genes modulated exclusively by the down-regulated miRNAs hsa-miR-143-3p and miR-205-5p, excluding the gene shared with hsa-106b-5p. There was only one target gene in common between hsa-miR-143-3p and miR-205-5p, while 51 target genes were overlapped between hsa-miR-21-5p and miR-106b-5p as listed in Figure 2B. We based the rest of the analysis on this 51-gene signature.

A.

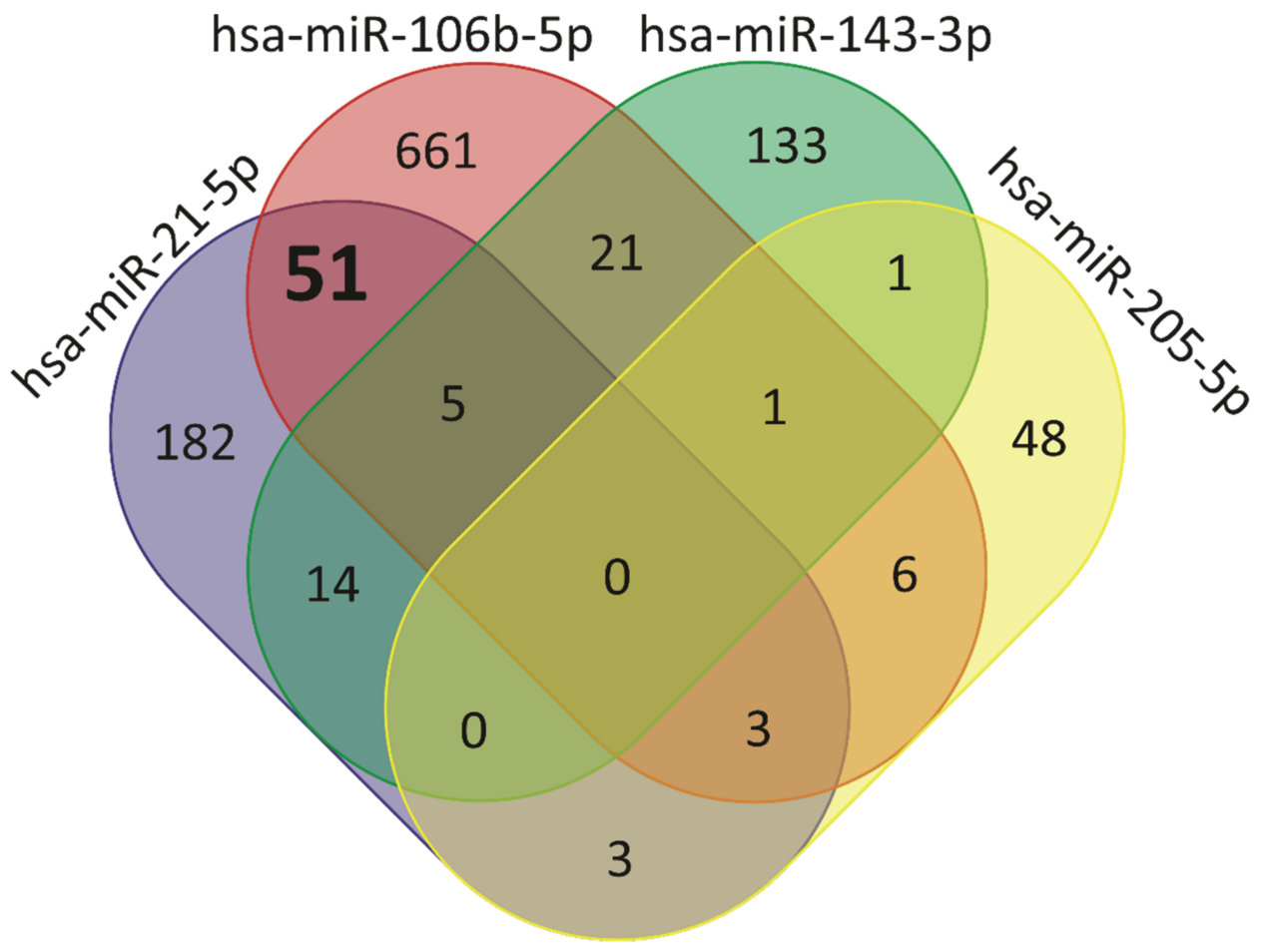

B.

\begin{tabular}{|c|c|c|c|c|c|}
\hline ABAT & CNOT6 & HBP1 & MEF2A & RPS6KA3 & UBE2W \\
\hline APPL1 & DEGS1 & HIAT1 & MSI2 & RSF1 & WEE1 \\
\hline ARL1 & EPHA4 & IGF2BP1 & NIPAL1 & RYBP & YOD1 \\
\hline ARMC8 & FAM160B1 & KANSL1L & PAFAH1B1 & SCD & YY1 \\
\hline ARRDC3 & FAM46C & KPNA4 & PANK3 & SESN1 & ZRANB1 \\
\hline BRWD1 & FRS2 & LAMC1 & PHF6 & SNX30 & ZYG11B \\
\hline CBFB & GAB1 & LTV1 & PLAG1 & STAT3 & \\
\hline CCNI & GAPVD1 & MAP3K2 & PPP1R3B & TBCEL & \\
\hline CHD9 & GNG12 & MARCH6 & PRRX1 & TOPORS & \\
\hline
\end{tabular}

Figure 2. Common target genes between hsa-miR-106b-5p and miR-21-5p. (A) The Venn diagram was performed using validated target genes from hsa-miR-106b-5p, miR-21-5p, miR-143-3p and miR-205-5p. (B) The 51 target genes shared exclusively between hsa-miR-106b-5p and miR-21-5p, but not with hsa-miR-143-3p and miR-205-5p, are listed.

\subsection{Hsa-miR-21-5p and miR-106b-5p Negatively Correlate with Their Target Genes}

In order to determine the relevance of hsa-miR-21-5p and miR-106b-5p in human tissues, we performed principal component analysis (PCA) of the 51 genes using normalized expression data from normal mammary gland (NMG) (GTEX), NAT and BCa samples 
(TCGA BRCA). The first two components (Dim 1 and 2), which explained $33.4 \%$ and 9.3\%, respectively, of the total variation, were plotted. We found marked differences in the overall gene expression between NMG and BCa tissues (Figure 3A, I). As shown in Figure 3A, II, the 51 genes contributed to Dim 1 in similar ways indicating that almost all the genes contributed to the discrimination between tumor and non-tumor tissues.

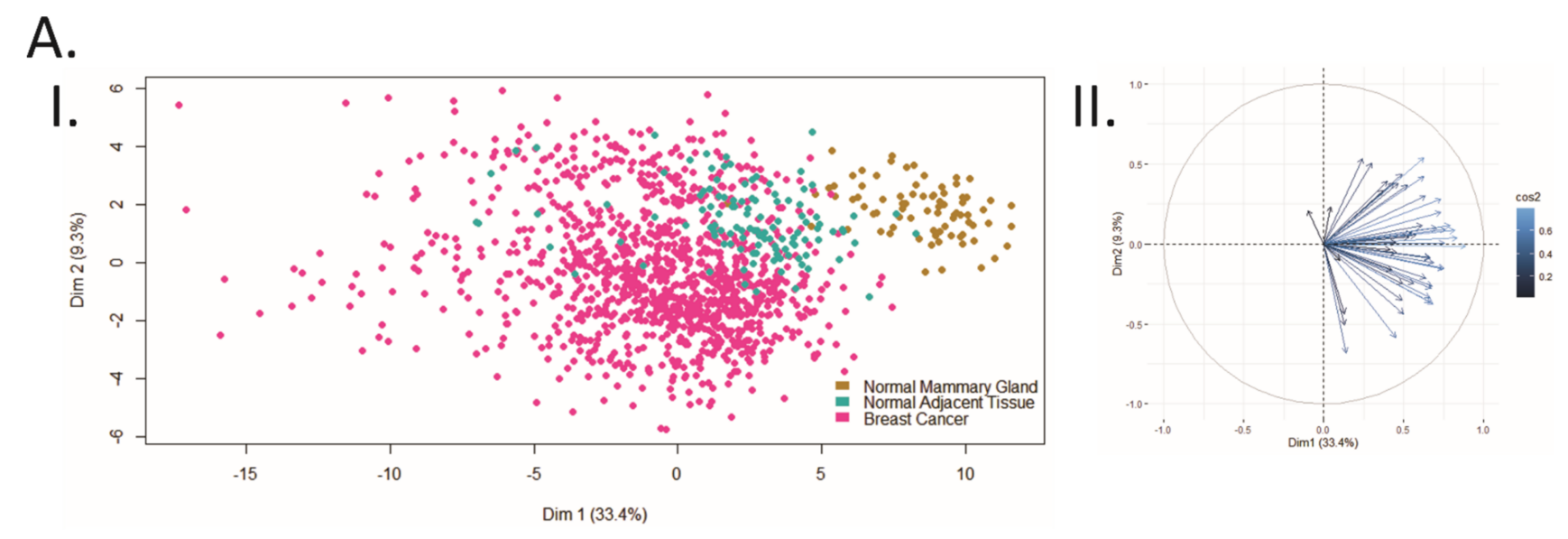

B.

BCa tissue
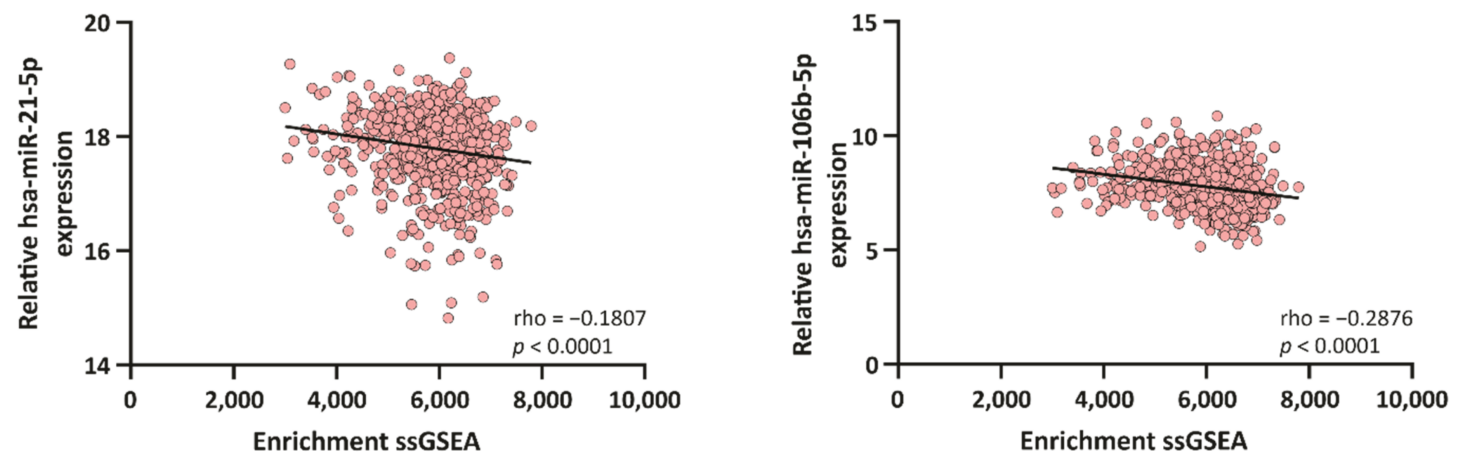

NAT tissue
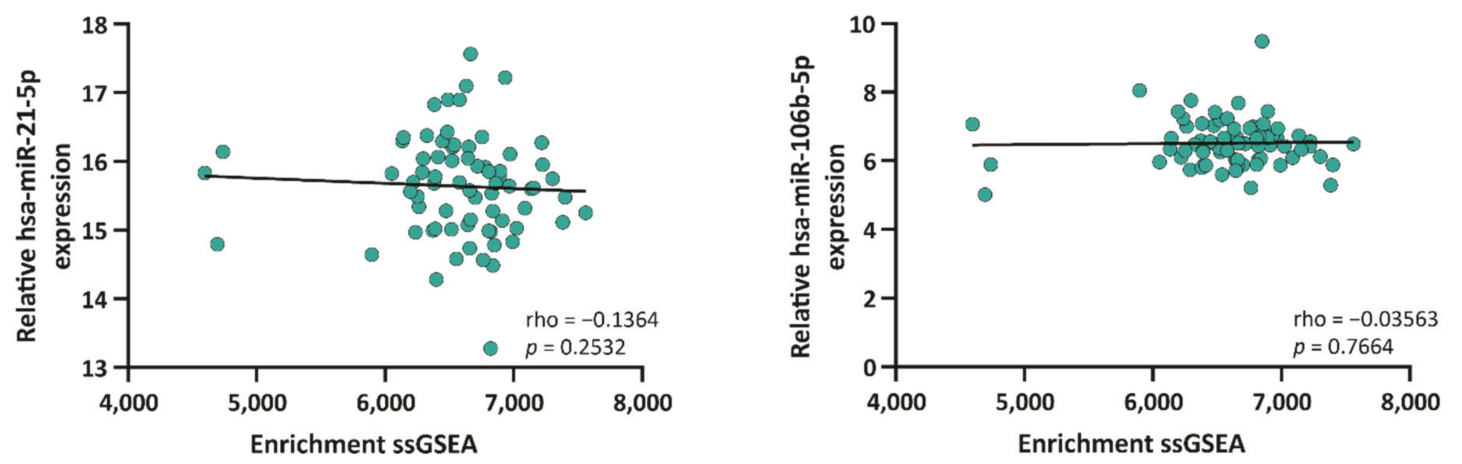

Figure 3. Principal component analysis showing correlation between hsa-miR-106b-5p and miR-21-5p with their target genes. (A) I. Scatterplot of the two principal components of principal component analysis (PCA) from the target genes expression data. The golden, blue and pink circles represent normal mammary gland (NMG), normal adjacent tissue (NAT) and BCa samples, respectively. II Biplot representing the common genes and their relevance in each dimension of the plot. (B) Spearman correlation between the ssGSEA enrichment scores calculated based on the 51 target genes and mature miRNA expression in BCa tissue and NAT from the TCGA-BRCA dataset. 
Additionally, we performed single sample gene set enrichment analysis (ssGSEA) to explore whether the 51-gene signature were coordinately up- or down-regulated within the BCa or the NAT samples using TCGA BRCA dataset. We found that the 51 target genes showed a positive ssGSEA-enrichment in BCa and NAT samples (Figure 3B). In addition, a negative correlation between the ssGSEA-enrichment and the expression of hsa-miR-21-5p and miR-106b-5p was found in BCa tissue (rho $=-0.1807$ and -0.2876 , respectively). These results suggest that hsa-miR-21-5p and miR-106b-5p might play a relevant role in the tumorigenesis, being able to distinguish normal from tumor tissue, based on their gene expression profile.

\subsection{Hsa-miR-21-5p and miR-106b-5p Modulate Cancer and Metabolic Related Pathways}

To further analyze the relevant pathways associated with the miRNA-target genes, we performed a KEGG pathway enrichment analysis $(p$-value $<0.05)$ for all the EVT genes of hsa-miR-21-5p, miR-106b-5p, miR-205-5p and miR-143-3p (Figure 4A, Tables S2 and S3). In particular, this analysis showed 12 common target genes, indicated with an asterisk at Figure 4A, between hsa-miR-21-5p and miR-106b-5p associated with processes related to cancer, including the MAPK signaling pathway, TGF- $\beta$ signaling pathway, ERBB1 downstream pathway, mTOR signaling pathway and Wnt signaling pathway; and several metabolic processes related to the insulin signaling pathway.

Moreover, analyzing the hsa-miR-205-5p and miR-143-3p target genes, we found several related cancer pathways, including senescence and autophagy, signaling by EGFR in cancer, microRNA regulation of DDR among others, but no genes were shared between these two miRNAs.

We next focused on the 12 genes indicated in Figure 4A: GAB1, GNG12, HBP1, MEF2A, PAFAH1B1, PPP1R3B, RPS6KA3, SESN1, MAP3K2, YY1, FRS2 and STAT3. We analyzed the expression of these genes in BCa and NAT tissue (Figure $4 \mathrm{~B}$ ). We found that GAB1, GNG12, HBP1, MEF2A, PAFAH1B1, PPP1R3B, RPS6KA3 and SESN1 were downregulated in BCa compared to NAT; meanwhile, $M A P 3 K 2$ and $Y Y 1$ were up-regulated. There were no statistical differences between BCa and NAT in FRS2 and STAT3. We continued the analysis with the eight target genes that were down-regulated in $\mathrm{BCa}$, as these could potentially be tumor suppressor genes.

2.5. Hsa-miR-106b-5p and miR-21-5p Are Upregulated in More Aggressive BCa Subtypes and Are Predictors of Worse Overall Survival

We evaluated the expression of hsa-miR-21-5p and miR-106b-5p in BCa from the TCGA BRCA dataset and compared them between subtypes and stages (Figure 5A). We found that hsa-miR-106b-5p was up-regulated in more aggressive subtypes (Basal), while hsa-miR-21-5p was up-regulated in intermediate aggressive subtypes (Luminal B and Her2). No significant differences were found in terms of the stage for these miRNAs.

Additionally, we performed Kaplan-Meier plots to evaluate the overall survival (OS) based on the expression of these miRNAs (Figure 5B) using a cohort of 1262 BCa patients. We found that patients who had a higher expression of hsa-miR-21 and miR-106b had lower survival and worse prognosis. Altogether these results propose hsa-miR-21-5p and miR-106b-5p as new biomarkers for BCa prognosis. 
A.

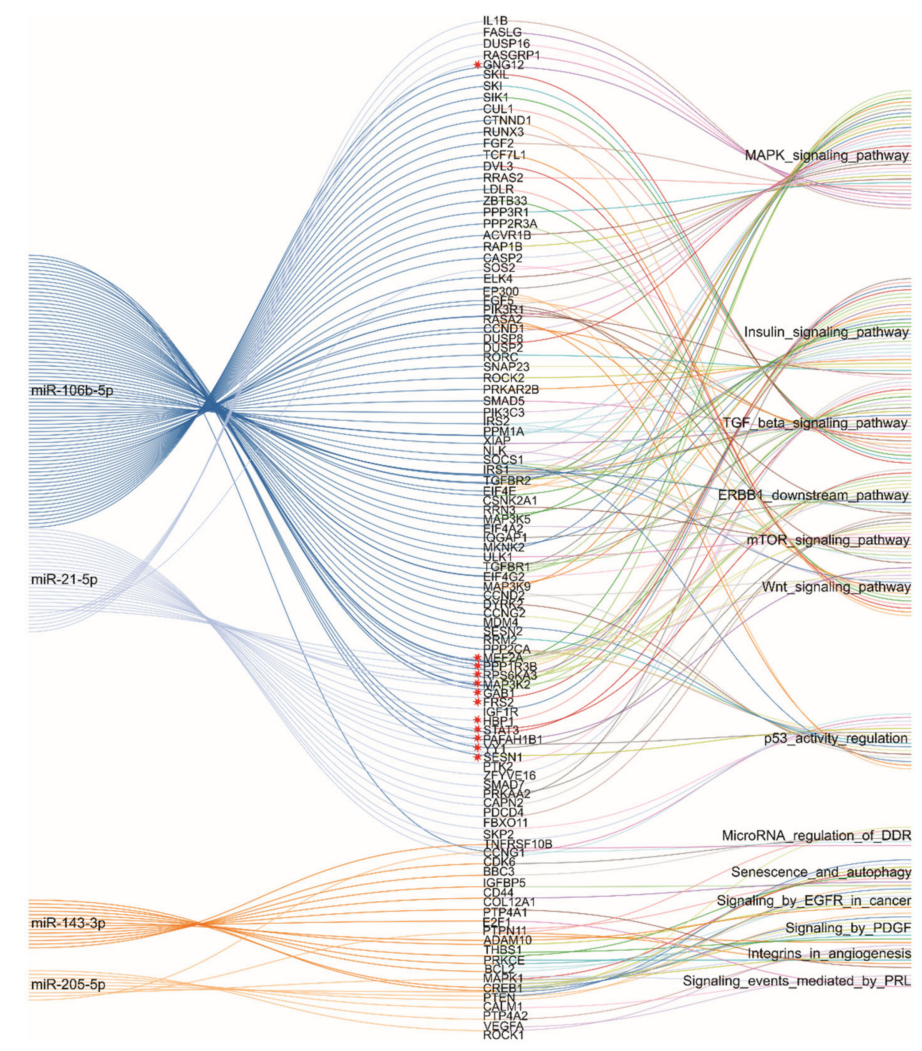

B.
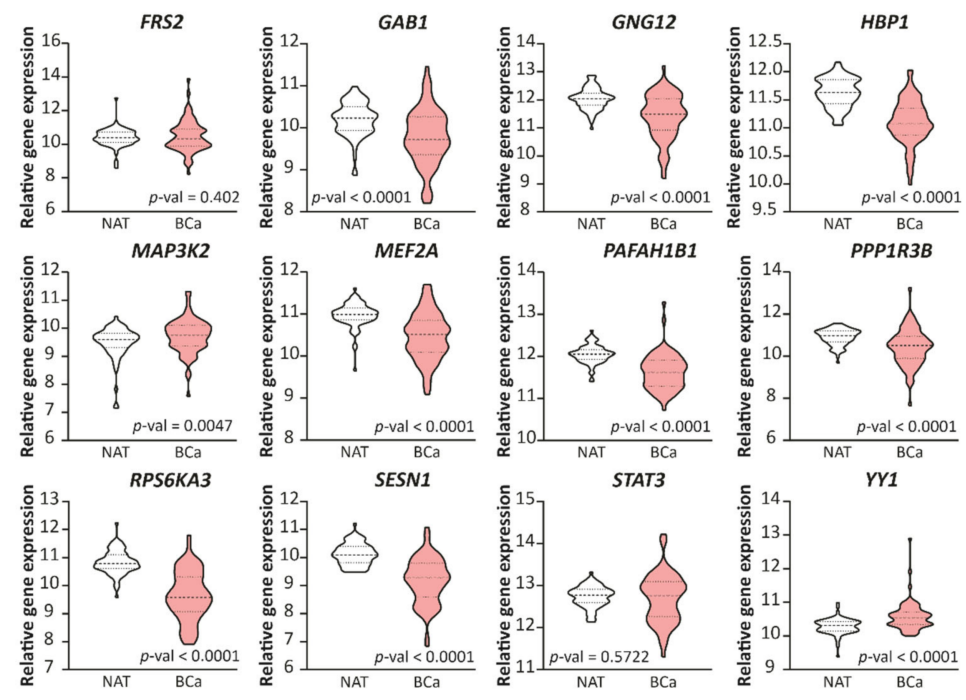

Figure 4. Functional enrichment of validated miRNA targets and their expression in human samples. (A) String diagram performed based on the top five significant KEGG pathways associated to the target genes of the up- and down-regulated miRNAs in common between humans and mice. Relevant genes related to the up-regulated miRNAs are marked with a red asterisk. (B) Expression levels of the selected genes in BCa tissue paired with normal adjacent tissue (NAT) from the TCGA-BRCA dataset are graphed in read per millions. The name of the genes is indicated up each plot in bold. Statistical analysis was performed using paired $T$-test or Wilcoxon test when corresponded. 
A.

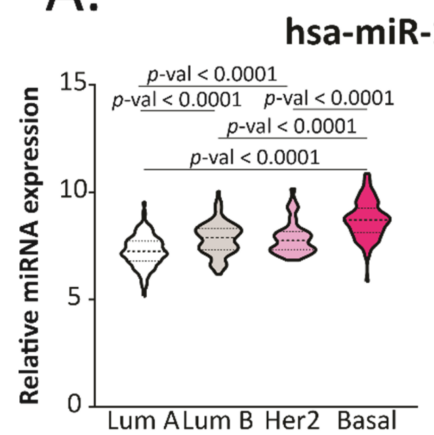

\section{-106b-5p}

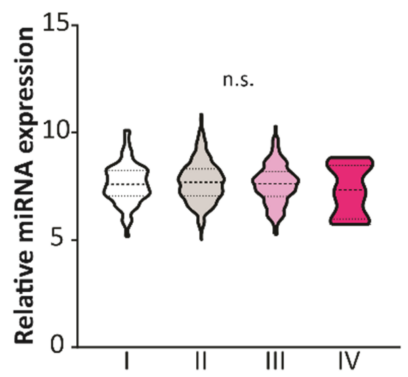

hsa-miR-21-5p
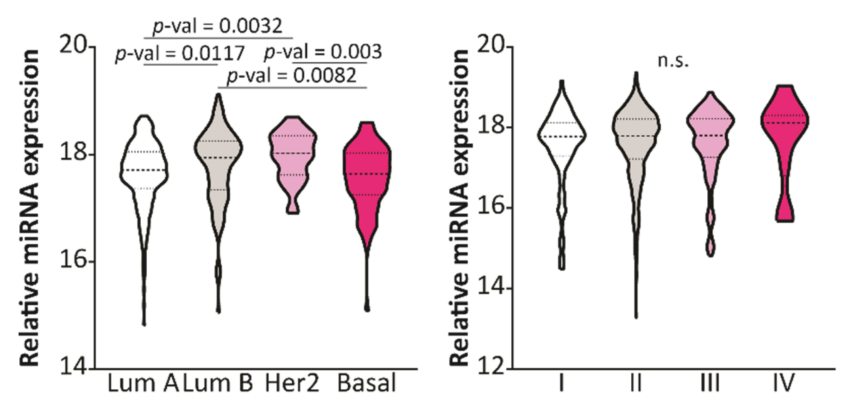

B.

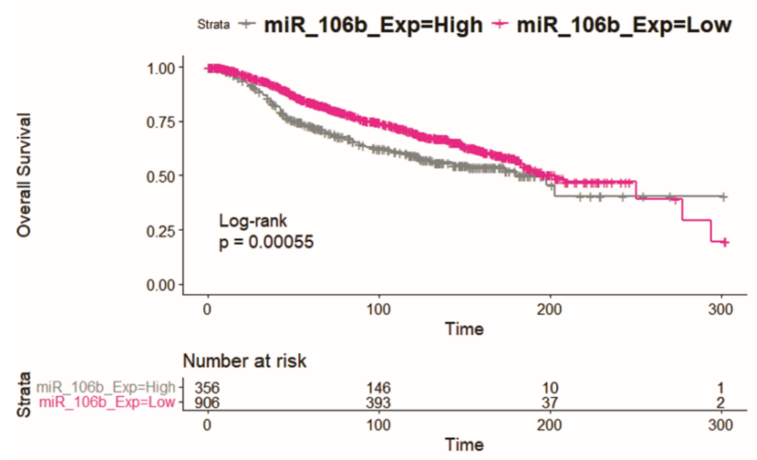

Strata + miR_21_Exp=High + miR_21_Exp=Low
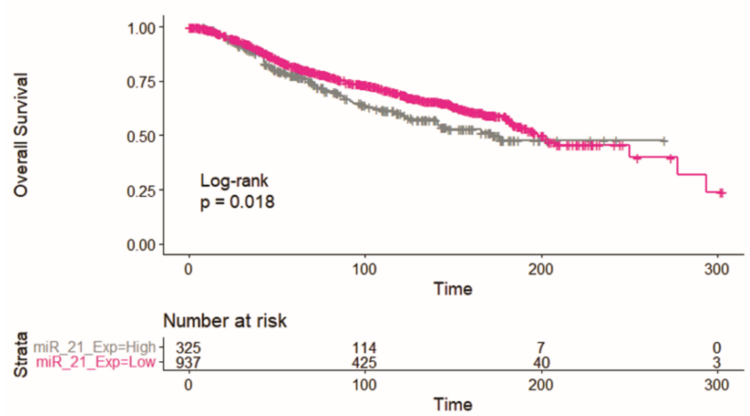

C.

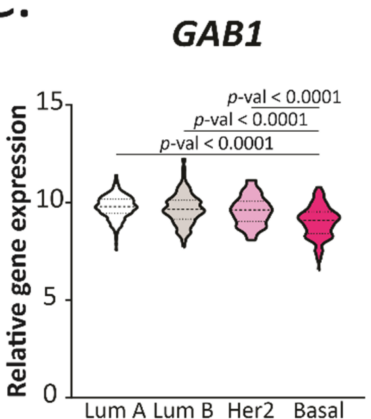

PAFAH1B1
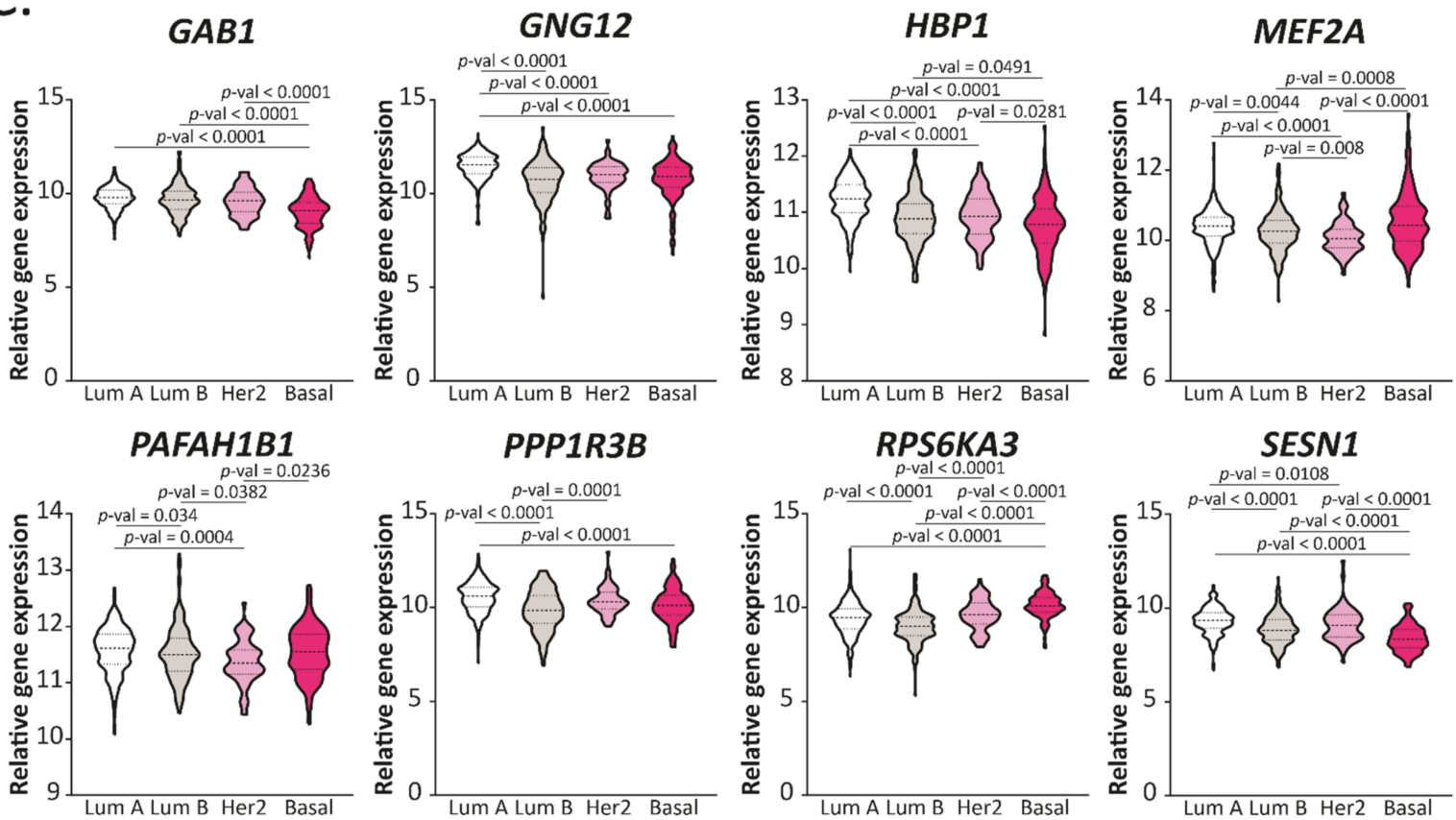

PPP1R3B

RPS6KA3
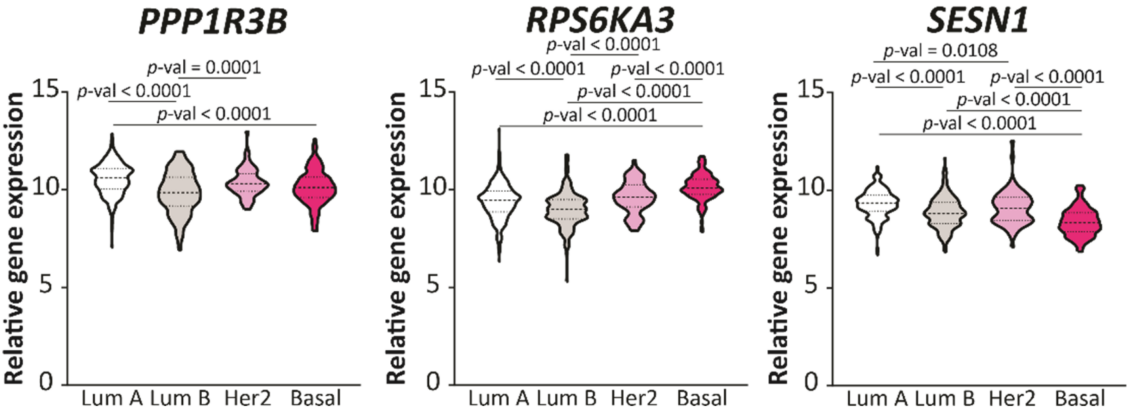

Figure 5. Hsa-miR-106b-5p, miR-21-5p and their target genes are deregulated in aggressive BCa subtypes and correlate with overall survival. (A) Expression levels of hsa-miR-106b-5p and miR-21-5p in BCa tissue divided in subtypes (Luminal A, Luminal B, Her2 or Basal) or Stages (I to IV) from the TCGA-BRCA dataset are graphed in read per millions. The name of the miRNAs is indicated up each plot in bold. Statistical analysis was performed using One-Way ANOVA or Kruskal-Wallis test followed by Tukey or Dunn's, respectively, when corresponded. (B) Kaplan-Meier curves comparing the overall survival (OS) between BCa patients with high or low expression of hsa-miR-106b-5p or miR-21-5p, respectively, in tumors. Statistical analysis was performed using the Cox-Mantel test. (C) Expression levels of the mentioned genes in BCa tissue divided in subtypes (Luminal A, Luminal B, Her2 or Basal) from the TCGA-BRCA dataset are graphed in read per millions. The name of the miRNAs is indicated up each plot in bold. Statistical analysis was performed using One-Way ANOVA or the Kruskal-Wallis test followed by Tukey or Dunn's, respectively, when corresponded. 
2.6. GAB1, GNG12, HBP1 and SESN1 Are Downregulated in More Aggressive BCa Subtypes and Could Be Used as Prognosis Biomarkers

We further analyzed the relevance of the eight selected miRNA-target genes from Figure $4 \mathrm{~B}$ in terms of aggressiveness based on their expression in different BCa tissue subtypes (Figure 5C). We found that GAB1, GNG12, HBP1 and SESN1 expression was reduced in more aggressive compared with less aggressive subtypes, in concordance to the fact that hsa-miR-106b-5p was up-regulated in these tissues and could be repressing these genes. Moreover, we found that MEF2A, PAFAH1B1, PPP1R3B and RPS6KA3 were downregulated in intermediate aggressive tumors (Luminal $B$ and Her2) and up-regulated in Luminal A and Basal subtypes, suggesting that these genes could be regulated mostly by hsa-miR-21-5p.

We also performed Kaplan-Meier plots to determine OS and relapse-free survival (RFS) from the genes that were down-regulated in more aggressive subtypes in a cohort of 1879 BCa patients. As shown in Figure 6, patients with low tumor expression of GNG12, HBP1 and SESN1 genes presented reduced OS (Figure 6A) and worse RFS (Figure 6B), compared to patients with high expression of these genes. Moreover, low GAB1 expression showed only worse RFS without changes in the OS. These results reinforce the relevant role for these genes as prognosis biomarkers in advanced BCa patients.

\subsection{GAB1, GNG12, HBP1 and SESN1 Negatively Correlate with hsa-miR-106b-5p in $B C a$ Tissues}

To determine whether GAB1, GNG12, HBP1 and SESN1 could be regulated by hsamiR-106b-5p in $B C a$ tissue, we performed a correlation matrix using available datasets in TCGA BRCA. We found that GAB1, GNG12, HBP1 and SESN1 negatively correlated with hsa-miR-106b-5p, with rho values of $-0.19,-0.38,-0.29$ and -0.31 , respectively (Figure 7A). Interestingly, GAB1, GNG12 and $H B P 1$ genes positively correlated with $H B P 1$ with rho values of $0.4,0.39$ and 0.35 , respectively (Figure 7A).

We plotted the expression of hsa-miR-106b-5p and each target gene to determine their correlation based on BCa subtype. As shown in Figure 7B, we found that more aggressive subtypes, such as Basal, had higher expression of hsa-miR-106b-5p and lower expression of the four target genes, in comparison with less aggressive subtypes, such as Luminal A, that had a lower expression of hsa-miR-106b-5p and higher expression of the target genes.

To determine the relevance of these genes in BCa metastasis, we used TNMplot in a cohort of 7893 subjects. We found that the expression of GAB1, GNG12, HBP1 and SESN1 was reduced in BCa compared to normal breast tissues (Figure 7C), as we previously showed in the TCGA cohort (Figure 4B). More importantly, these genes' expression was dramatically diminished in metastatic tissues compared to normal breast tissues and $\mathrm{BCa}$ (Figure 7C). 
A.
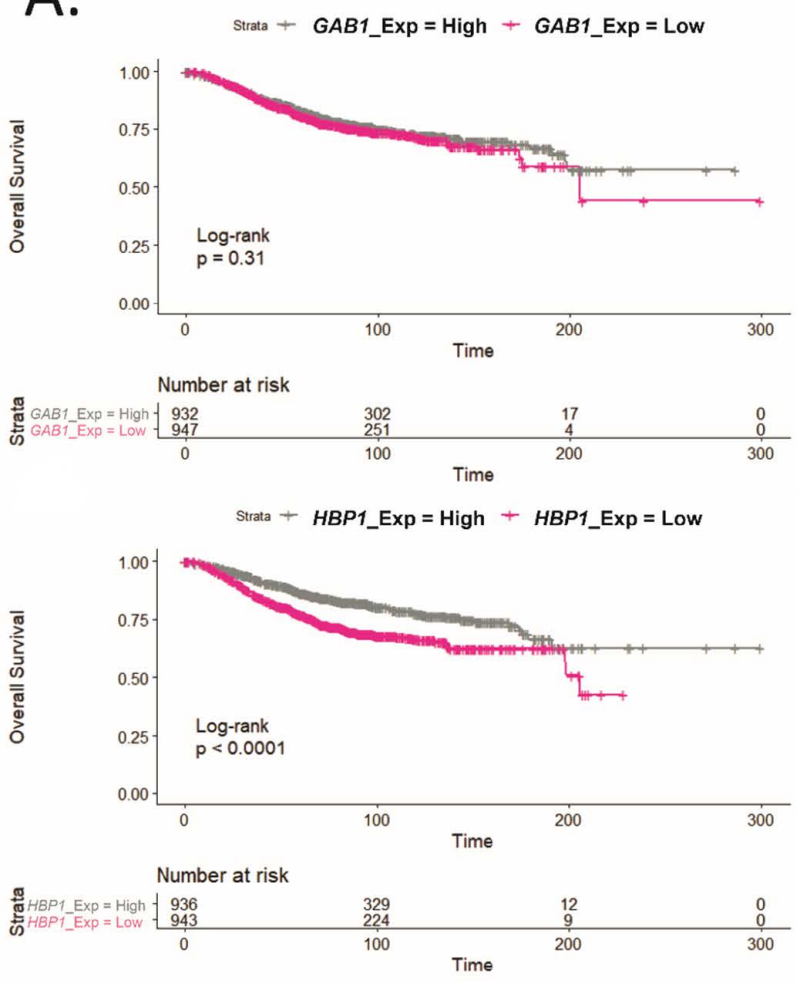

B.
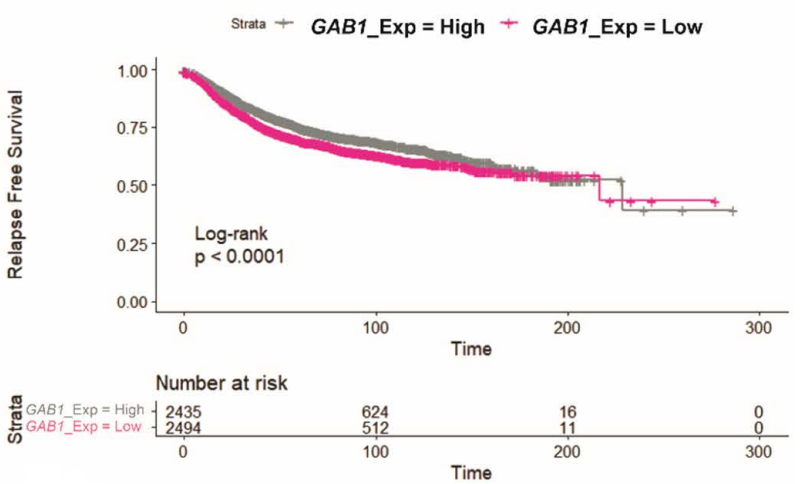

Strata + HBP1_Exp $=$ High + HBP1_Exp $=$ Low

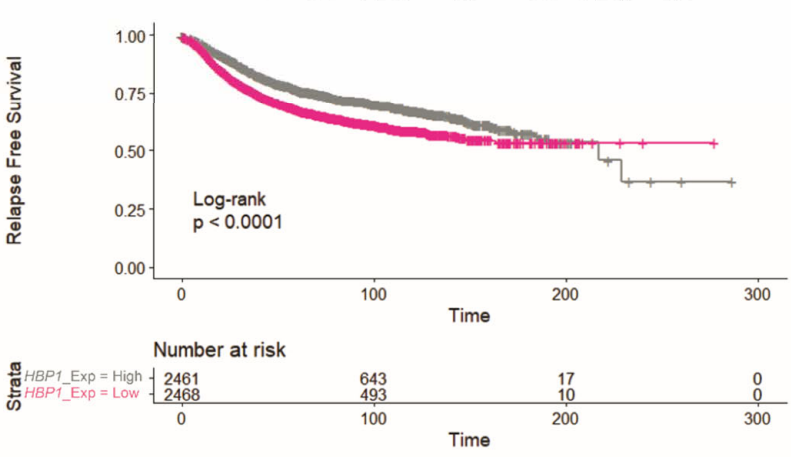

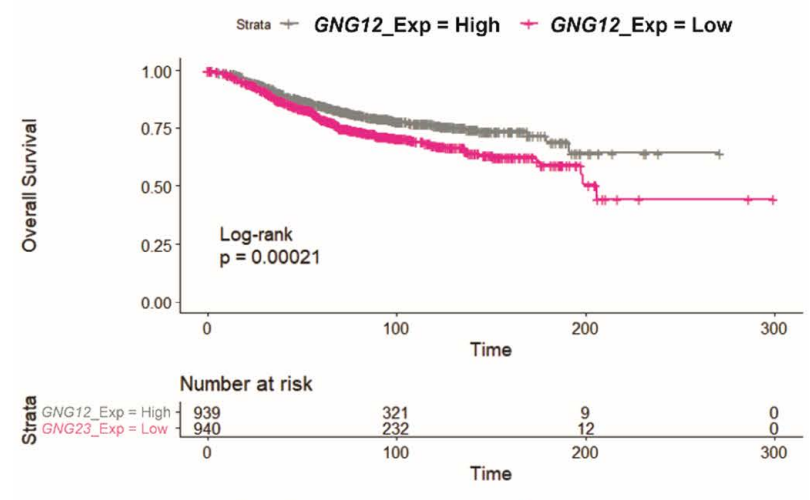
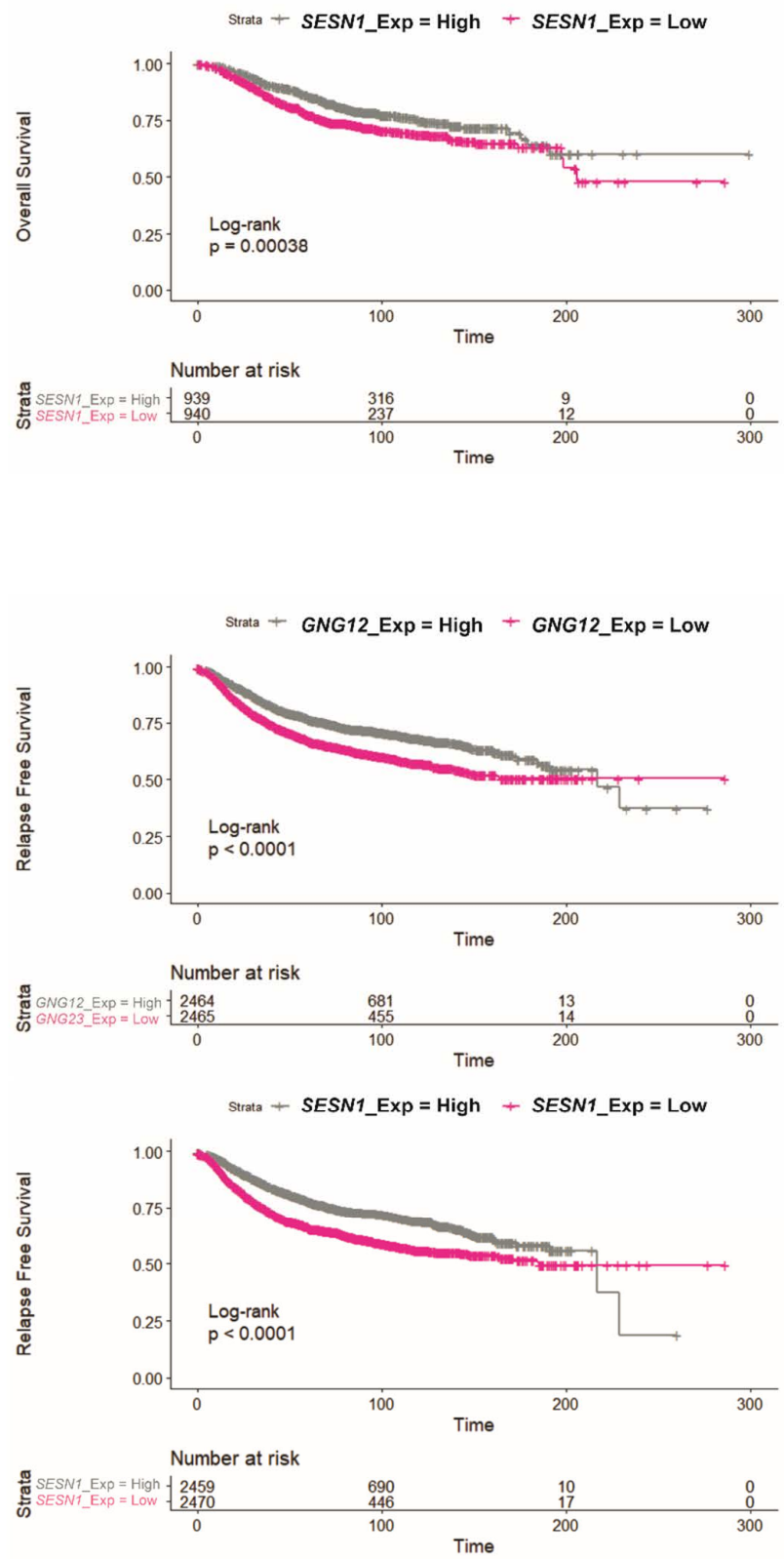

Figure 6. Lower expression of GAB1, GNG12, HBP1 and SESN1 correlates with worse relapse free survival and with overall survival. (A) Kaplan-Meier curve comparing the overall survival (OS) between BCa patients with high or low expression of the mentioned genes in tumors. Statistical analysis was performed using the Cox-Mantel test. (B) Kaplan-Meier curve comparing relapse free survival (RFS) between BCa patients with high or low expression of the mentioned genes in tumors. Statistical analysis was performed using the Cox-Mantel test. 


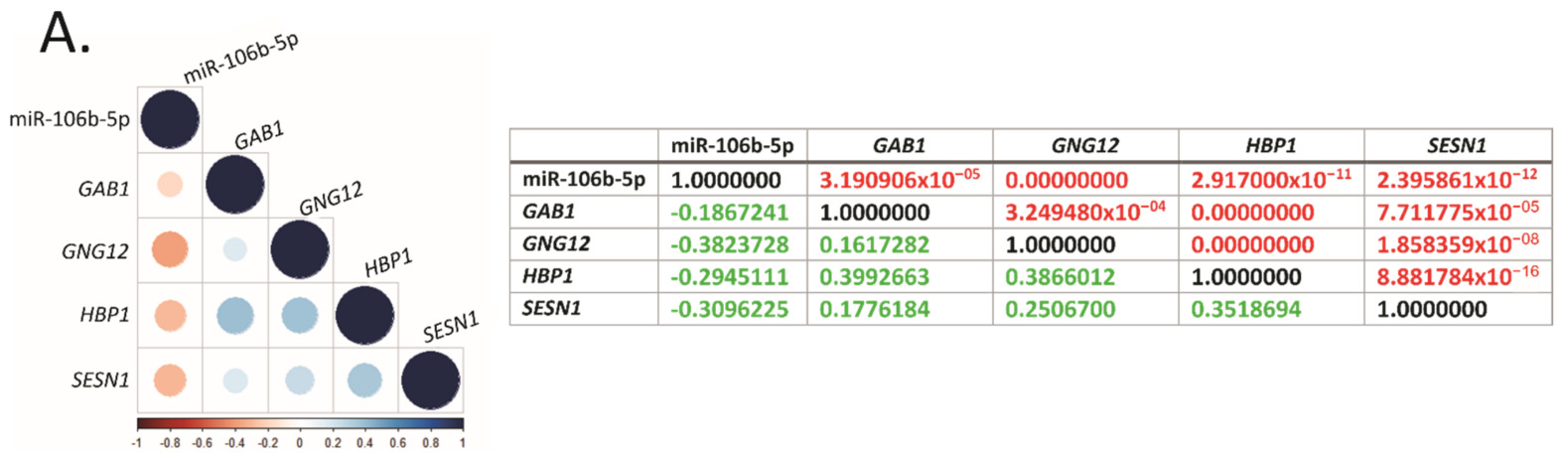

B.
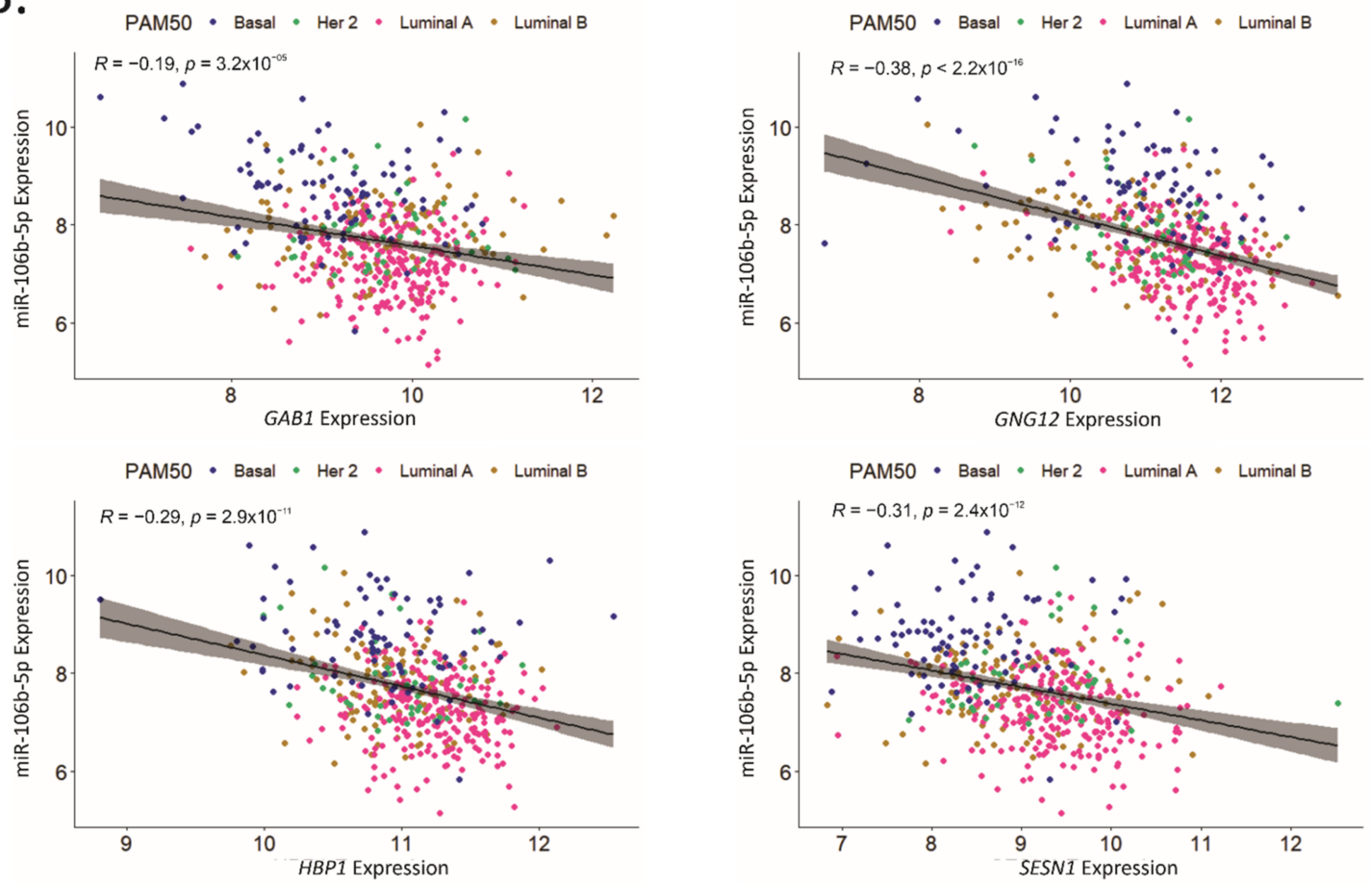

C.
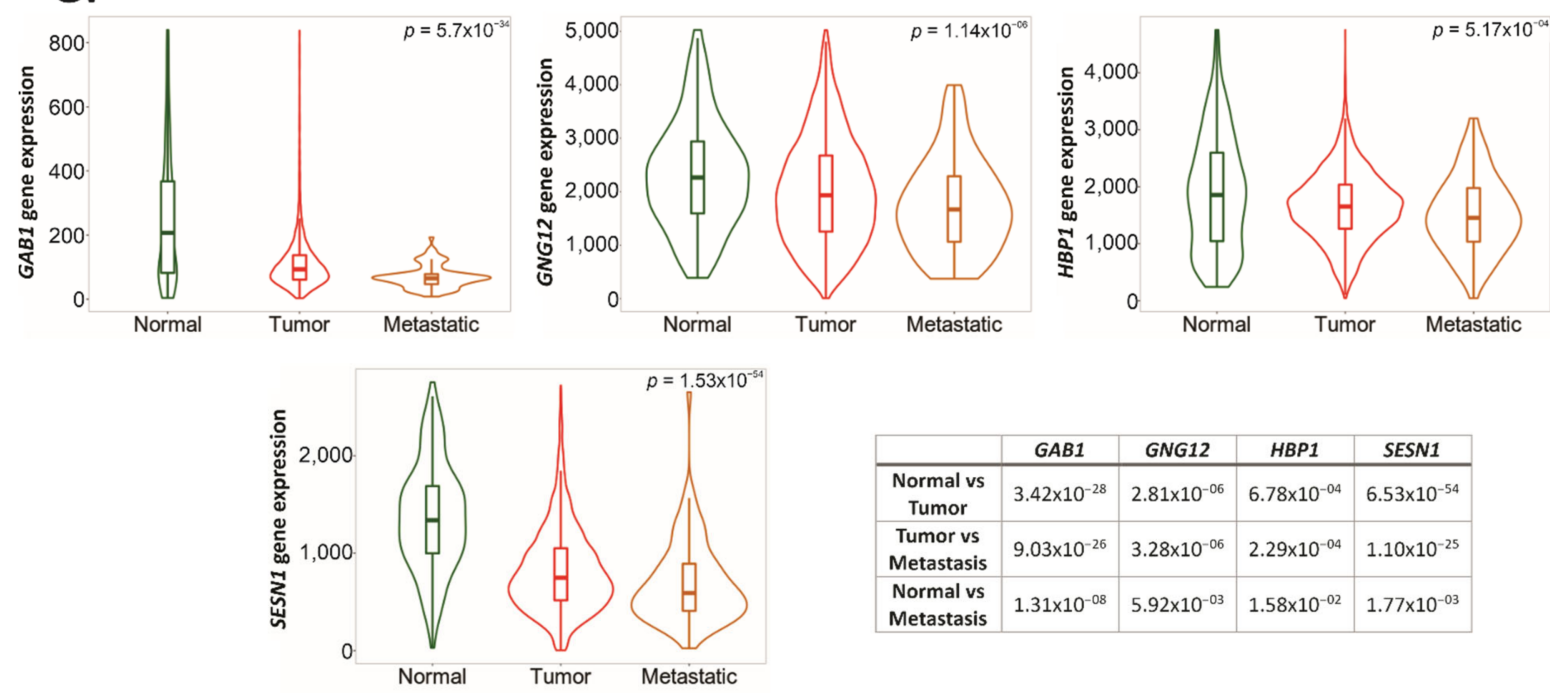

\begin{tabular}{|c|c|c|c|c|}
\hline & GAB1 & GNG12 & HBP1 & SESN1 \\
\hline $\begin{array}{c}\text { Normal vs } \\
\text { Tumor }\end{array}$ & $3.42 \times 10^{-28}$ & $2.81 \times 10^{-06}$ & $6.78 \times 10^{-04}$ & $6.53 \times 10^{-54}$ \\
\hline $\begin{array}{c}\text { Tumor vs } \\
\text { Metastasis }\end{array}$ & $9.03 \times 10^{-26}$ & $3.28 \times 10^{-06}$ & $2.29 \times 10^{-04}$ & $1.10 \times 10^{-25}$ \\
\hline $\begin{array}{c}\text { Normal vs } \\
\text { Metastasis }\end{array}$ & $1.31 \times 10^{-08}$ & $5.92 \times 10^{-03}$ & $1.58 \times 10^{-02}$ & $1.77 \times 10^{-03}$ \\
\hline
\end{tabular}

Figure 7. Hsa-miR-106b-5p negatively correlates with GAB1, GNG12, HBP1 and SESN1. (A) Matrix correlation showing existing correlation between hsa-miR-106b-5p expression in BCa tissue from the TCGA-BRCA cohort and target genes. $p$-values and Spearman correlation value are indicated in the table in red and green, respectively. (B) Spearman correlation between hsa-miR-106b-5p expression in BCa tissue from the TCGA-BRCA dataset, and each target gene is plotted 
distinguishing between BCa subtypes. The pink, golden, green and blue circles represent Luminal A, Luminal B, Her2 and Basal BCa subtypes, respectively. (C) Expression levels of the mentioned genes in normal mammary glands (Normal), BCa tissue (Tumor) and metastatic BCa (Metastatic) are graphed using TNMplot bioinformatic tool. Statistical analysis was performed using the Kruskal-Wallis (K-W) test followed by Dunn's. The p-value of the K-W test is indicated on the top right corner of each graph. Dunn's comparison's $p$-value corresponding to each gene and each graph are resumed in the table.

\section{Discussion}

In this work, we provide new evidence of miRNAs and genes that could be used in the study of BCa aggressiveness and prognosis. We found that hsa-miR-21-5p and miR-106b-5p were both up-regulated in $\mathrm{BCa}$ tissue in mice and human samples. Accordingly, eight miRNA target-genes (GAB1, GNG12, HBP1, MEF2A, PAFAH1B1, PPP1R3B, RPS6KA3 and $S E S N 1$ ) were downregulated in BCa compared to NAT tissue in patients.

We found that hsa-miR-21-5p was up-regulated in BCa subtypes Her2+, correlated with worse OS and could be regulating MEF2A, PAFAH1B1, PPP1R3B and RPS6KA3, since they were mostly downregulated in Her2+ BCa subtypes. Moreover, we found that hsa-miR-106b-5p was up-regulated in more aggressive BCa subtypes and correlated with worse OS, and four of the eight target genes (GAB1, GNG12, HBP1 and SESN1) were downregulated in more aggressive subtypes and correlated with worse OS and RFS. Finally, we found that their expression negatively correlated with hsa-miR-106b-5p expression in $\mathrm{BCa}$ tissue, proposing them as promising prognosis biomarkers.

There is compelling evidence proposing hsa-miR-21-5p as an oncomiR [17-20]. It is located in 17q23.2, which is a region that has been found to be amplified in breast carcinomas and contains several oncogenes [21]. This miRNA was found up-regulated in ductal carcinomas in situ (DCIS) compared to non-malignant breast tissues [21], in several cancers, including $\mathrm{BCa}[17,19,22,23]$ and in the circulation of BCa patients compared to healthy donors [21-24]. Moreover, it was found up-regulated in plasma from BCa patients with Her2 and Luminal B subtypes, which proposes a link between hsa-miR-21-5p and the Her2 receptor [25]. In particular, there is evidence of Her2 and hsa-miR-21-5p overexpression correlation, since hsa-miR-21-5p is up-regulated in Her2+ BCa [26], and it was also found associated to more aggressive BCa [20,27].

In terms of $\mathrm{BCa}$ progression, it was found to be related to $\mathrm{BC}$ a pathogenesis, proliferation, invasion, apoptosis, the epithelial to mesenchymal transition (EMT), cell cycle control and metastasis in tumor cells $[18-20,22,23,25,26]$. It was found an association between up-regulation of hsa-miR-21-5p and poor disease-free survival (DFS) in early stage BCa [20]; however, there is contradictory evidence for the correlation between hsa-miR-21-5p expression and OS $[17,18]$. Thus, these data, together with our findings, strongly support hsa-miR-21-5p as a biomarker for BCa diagnosis and prognosis.

Hsa-miR-106b-5p is a member of the 106b-25 cluster and a paralogous of the 17-92 cluster [28] that was found amplified and/or overexpressed in several tumors, including $\mathrm{BCa}$ [29-31]. It was up-regulated in the circulation of BCa patients compared to healthy donors [30] and in $\mathrm{BCa}$ compared to benign lesions [28,30]. In concordance to our findings, hsa-miR-106b-5p was found up-regulated in triple negative BCa compared to other subtypes [28]. The role of hsa-miR-106b-5p in BCa progression is highly reported: it was found associated to EMT [29,30,32-34], tumor progression [35], cell proliferation and migration [33,34], cell cycle control and cellular response to stress $[30,36]$ and apoptosis and angiogenesis $[29,31]$.

Preclinical studies demonstrated that hsa-miR-106b-5p can predict the presence of metastasis $[30,36]$ since mice with up-regulation of this miRNA in tumors had developed lung metastasis [34] and increased number of metastatic nodules in the liver [35]. Moreover, hsa-miR-106b-5p was found up-regulated in metastatic primary tumors compared to non-metastatic, up-regulated in metastasis compared to primary tumors [35] and even up-regulated in secondary metastasis compared to primary metastasis [36].

In terms of BCa patient survival, it has been reported that those who have lower hsamiR-106b-5p expression have longer DFS [35]. Moreover, in another study, BCa patients 
with higher expression of this miRNA had shorter DFS and OS as well as an augmented recurrence risk [30]. Higher levels of hsa-miR-106-5p were also associated to decreased tumor relapse [31]. In summary, all this evidence suggests hsa-miR-106-5p as a good biomarker to predict recurrence and progression and as a prognostic biomarker to identify local or distant recurrence and high-risk BCa.

In this work, we found that hsa-miR-106b-5p and miR-21-5p share several target genes, including GAB1, GNG12, HBP1 and SESN1, that were down-regulated in breast primary tumors and metastases. According to Lee et al. [37], GNG12 was found down-regulated in local recurrent DCIS compared to no recurrent, which strongly supports our data.

Furthermore, SESN1 was found to be mainly involved in metabolism regulation and aging via the AMPK/mTOR pathway [38]. In particular, it interacts with AMPK in the stress response, tumorigenesis suppression and maintenance of genomic integrity [38]. It was found that mutant p53 blockades SESN1 / AMPK complex, unleashing oncogenic effects such as inhibition of apoptosis, increasing drug resistance and proliferation [39]. It was found that, in patients with mutant p53, a lower expression of several genes, including SESN1, correlated with lower RFS times and distant-metastasis-free survival [40].

GAB1's role in BCa has been largely studied and, in opposition with our findings, it has been proposed as an oncogene. GAB1 is a key scaffold protein that enhances the downstream signaling of c-Met among others tyrosine-kinase receptors [41,42], it is involved in the formation of invadopodia [41], allows epidermal growth factor receptor (EGFR) dimers to directly signal PI3K [43] and is implicated in EGF-induced activation of MAPK [44].

In opposition with our findings, GAB1 was found up-regulated in breast tumors compared to benign mammary hyperplasia from patients [45]. Moreover, GAB1 overexpression was associated to metastasis in Her2 and triple negative BCa subtypes [45]. However, this study was limited to a low number of subjects, which could be the cause of disagreement with our results.

Several studies have proposed $H B P 1$ as a transcriptional repressor, since it has an HMG box DNA-binding domain that allows it to impair the union between DNA and active transcription factors [46-49]. HBP1 is located in chromosome 7q31.1, a region that is found usually deleted in several cancer types $[46,50]$ and $30 \%$ of BCa patients have mutants or variants of $H B P 1[47,48]$. In BCa preclinical models, HBP1 depletion increased cell cycle progression, proliferation, invasion, migration, and tumorigenesis $[47,49]$.

Additionally, HBP1 was found down-regulated in breast tumors compared to normal tissue from patients, which was associated with poor prognosis, RFS and relapse $[46,47,50]$. Furthermore, hsa-miR-17-5p, a member of the 17-92 cluster, paralogous of the 106b-25 cluster, binds to $H B P 1$, lowering its expression [50]. These findings could indicate that hsa-miR-106b-5p could also be involved in HBP1 decrease; however, further studies are needed to confirm our hypothesis.

In summary, all these data suggest that GAB1, GNG12, HBP1 and SESN1 act as tumor suppressor genes by the proposed mechanism indicated in Figure 8. As shown, less aggressive BCa subtypes and NAT display low expression of hsa-miR-106b-5p, which, in turn, maintains GAB1, GNG12, HBP1 and SESN1 high expression. However, more aggressive $\mathrm{BCa}$ correlates with hsa-miR-106b-5p up-regulation and the down-regulation of its targets GAB1, GNG12, HPB1 and SESN1, unleashing BCa aggressiveness and, therefore, worsening the prognosis of $\mathrm{BCa}$ patients. 


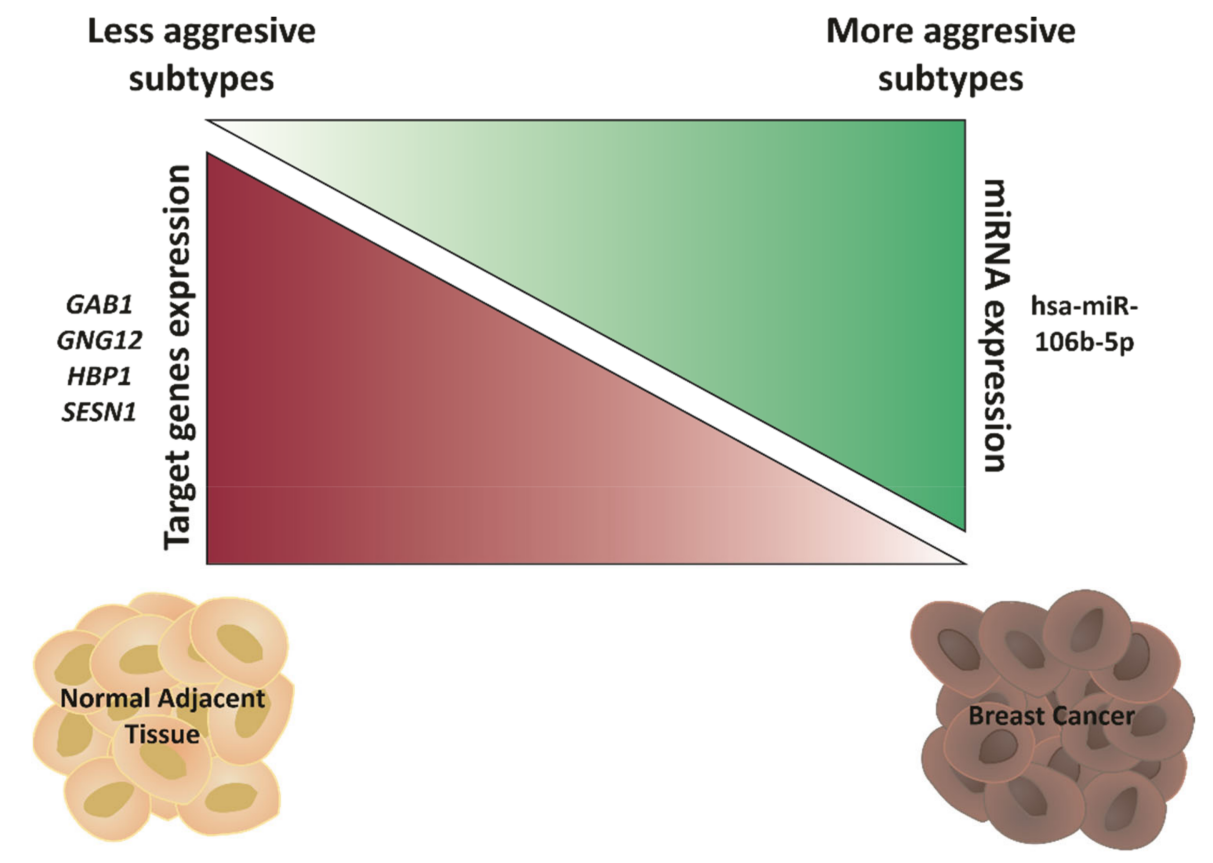

Figure 8. Hypothetical model. Hsa-miR-106b-5p is down-regulated in less aggressive BCa subtypes and NAT, which keeps GAB1, GNG12, HBP1 and SESN1 up-regulated. When BCa progresses, or in more aggressive BCa subtypes, hsa-miR-106b-5p is then up-regulated and targets GAB1, GNG12, HPB1 and SESN1, diminishing their expression, unleashing BCa aggressiveness and, therefore, resulting in a worse $\mathrm{BCa}$ prognosis.

\section{Materials and Methods}

\subsection{Cell Culture}

The 4T1 murine cell line (ATCC: CRL-2539) was cultured in RPMI 1640 (Invitrogen) supplemented with $10 \%$ of fetal bovine serum and antibiotics in a $5 \% \mathrm{CO}_{2}$ humidified atmosphere at $37^{\circ} \mathrm{C}$.

\subsection{BCa Allograft Murine Model}

We housed 16-week-old Balb/c female mice $(n=16)$ under pathogen-free conditions following the IBYME's animal care guidelines. Mice were randomized into two groups: Control and BCa. BCa mice were inoculated in the mammary fat pad with $1 \times 10^{4} 4 \mathrm{~T} 1$ cells. Control animals were not inoculated at any point of the experiment. Control and BCa were sacrificed both at the same time (4 weeks after the inoculation) and tumor and mammary gland (MG) samples were collected. Tumor measurement was done as previously described [51].

\subsection{RNA Isolation and RT-qPCR Analysis}

The total RNA from allografts and MG were isolated using TriReagent (Molecular Research Center). Stem-loop RT-qPCR method was used to retrotranscribe miRNAs as previously described [52-54]. Briefly, $100 \mathrm{ng}$ of total RNA and $0.07 \mu \mathrm{M}$ of stem-loop specific primer were preheated at $70^{\circ} \mathrm{C}$ for $5 \mathrm{~min}$. Then, retrotranscription was performed using M-MLV RT (Promega) and incubated in TC 9639 Thermal Cycler (Benchmark) at $16^{\circ} \mathrm{C}$ for $30 \mathrm{~min}, 42{ }^{\circ} \mathrm{C}$ for $60 \mathrm{~min}$ and $70{ }^{\circ} \mathrm{C}$ for $2 \mathrm{~min}$. qPCRs were performed in StepOne Plus Real Time PCR (Applied Biosystems) using Taq DNA Polymerase (Pegasus) as previously described [51].

All reactions were run in duplicate. The expression levels of miRNAs were calculated using the $\Delta \Delta \mathrm{Ct}$ method normalizing to mmu-miR-191-5p and the Control. The primer sequences for Stem-loop RT-qPCR are listed in Table 1. The results are given as the median and interquartile ranges. Data normalization and homogeneity of variances was assessed 
using the Shapiro-Wilk test, F test or boxplot, respectively. Student's $t$-test was applied for data that fulfill the requirements. Otherwise, the Welch, Wilcoxon or Median test was performed. We used a significance level of $5 \%$.

Table 1. Primer sequences used for stem-loop RT-qPCR.

\begin{tabular}{|c|c|c|}
\hline Primer & Sequence $\left(5^{\prime}-3^{\prime}\right)$ & $\mathrm{T}_{\text {ann }}\left({ }^{\circ} \mathrm{C}\right)$ \\
\hline RT-Stem-loop-Rv & TGGTGCAGGGTCCGAGGTATT & - \\
\hline RT-mmu-miR-21a-5p-STEM & GTCTCCTCTGGTGCAGGGTCCGAGGTATTCGCACCAGAGGAGACTCAACA & - \\
\hline RT-mmu-miR-21a-5p Fw & CGGGGGGTAGCTTATCAGACTG & 65 \\
\hline RT-mmu-miR-106b-5p-STEM & GTCTCCTCTGGTGCAGGGTCCGAGGTATTCGCACCAGAGGAGACATCTGC & - \\
\hline RT-mmu-miR-106b-5p Fw & GCGGCGGTAAAGTGCTGACAG & 67 \\
\hline RT-mmu-miR-125b-5p-STEM & GTCTCCTCTGGTGCAGGGTCCGAGGTATTCGCACCAGAGGAGACTCACAA & - \\
\hline RT-mmu-miR-125b-5p Fw & CCGCСТCССТGAGACССТAAC & 65 \\
\hline RT-mmu-miR-221-3p-STEM & GTCTCСТCTGGTGCAGGGTCCGAGGTATTCGCACCAGAGGAGACGAAACC & - \\
\hline RT-mmu-miR-221-3p Fw & GGCGGAGCTACATTGTCTGCTG & 65 \\
\hline RT-mmu-miR-138-5p-STEM & GTCTCCTCTGGTGCAGGGTCCGAGGTATTCGCACCAGAGGAGACCGGCCT & - \\
\hline RT-mmu-miR-138-5p Fw & GGCGGAGCTGGTGTTGTGAATC & 67 \\
\hline RT-mmu-miR-143-3p-STEM & GTCTCСTCTGGTGCAGGGTCCGAGGTATTCGCACCAGAGGAGACGAGCTA & - \\
\hline RT-mmu-miR-143-3p Fw & GGGCGGTGAGATGAAGCACTG & 67 \\
\hline RT-mmu-miR-146a-5p-STEM & GTCTCCTCTGGTGCAGGGTCCGAGGTATTCGCACCAGAGGAGACAACCCA & - \\
\hline RT-mmu-miR-146a-5p Fw & CGGGCGGTGAGAACTGAATTCC & 65 \\
\hline RT-mmu-miR-205-5p-STEM & GTCTCCTCTGGTGCAGGGTCCGAGGTATTCGCACCAGAGGAGACCAGACT & - \\
\hline RT-mmu-miR-205-5p Fw & CGCGTCCTTCATTCCACCGG & 65 \\
\hline RT-mmu-miR-191-5p-STEM & GTCTCCTCTGGTGCAGGGTCCGAGGTATTCGCACCAGAGGAGACCAGCTG & - \\
\hline RT-mmu-miR-191-5p Fw & GCGGCAACGGAATCCCAAAAG & 70 \\
\hline
\end{tabular}

\subsection{TCGA Dataset Analysis}

Clinical-pathological data, mature miRNA and gene expression of breast cancer (BCa) $(n=1097)$ and normal adjacent tissue (NAT) $(n=114)$ of patients, and normal mammary gland (NMG) $(n=80)$ was obtained from de TCGA Breast Cancer (BRCA) cohort or from the GTEx project available in the UCSC Xena bioinformatics tool (https:/ / xena.ucsc.edu/, accessed on 15 August 2021) [16]. The miRNA-Seq (IlluminaHiSeq_miRNASeq) and RNASeq (IlluminaHiSeq) data was downloaded as $\log _{2}(R P M+1)$ values. Seventy-five BCa samples paired with 75 NAT were included in the present study.

When miRNAs were analyzed, 491 BCa samples were used, and, when genes were analyzed, 821 BCa samples were used. Data normalization and homogeneity of variances was assessed using the Shapiro-Wilk test, and Levene, F test or boxplot, respectively. A paired sample $t$-test was applied for data that fulfill the requirements. Otherwise, the Wilcoxon test was performed. When three or more groups were analyzed, one-way ANOVA followed by Tukey was performed for data that fulfill the requirements. Otherwise, the Kruskal-Wallis followed by Dunn's Test was performed. We used a significance level of $5 \%$.

\subsection{Functional Enrichment Analysis}

To identify experimentally validated target (EVT) genes regulated by differentially expressed miRNAs in human BCa and mice allografts selected miRNAs, we employed the DIANA TARBASE v8 resource (threshold score $>0.5$ ) [55]. Functional enrichment analyses of the obtained gene lists were performed using the ClueGo Cytoscpape's plug in and the Enrichr resource. For pathway terms and annotation, we used those provided by KEGG and BioPlanet (http:/ / tripod.nih.gov/bioplanet/, accessed on 1 August 2021; https: / / www.genome.jp/kegg/pathway.html, accessed on 1 August 2021). For network construction of the interactions miRNA-target-pathways, we used a Sankey diagram.

\subsection{Principal Component Analysis (PCA)}

Publicly available gene expression values for TCGA Breast Cancer (BRCA) and the GTEx project patient samples were downloaded as previously described. PCA was performed to determine samples distribution based on the expression of 51 common target 
genes from up-regulated miRNAs in both breast human tissue and mice allografts. For PCA plots, the R function "prcomp" from stats package (version 4.0.2) was used.

\subsection{Single-Sample Gene-Set Enrichment Analysis}

A ssGSEA was performed to analyze the coordinated regulation of a defined gene signature and the activation of specific biological processes in BCa samples. $\log _{2}(\mathrm{RPM}+1)$ gene expression values of $474 \mathrm{BCa}$ samples were obtained from TCGA-BRCA cohort and loaded into GenePattern web-tool (https: / / www.genepattern.org, accessed on 30 August 2021). A gene set enrichment profile was obtained for each sample using a 51 gene signature obtained from common target genes from up-regulated miRNAs in both breast human tissue and mice allografts. Then, Spearman correlation analysis was performed between the expression of two up-regulated miRNAs and the enrichment score obtained from the ssGSEA (GraphPad Prism 8.0.1; Prism, San Diego, CA, USA).

\subsection{Correlation Matrix}

Expression levels of miRNA and target genes in breast tumors from patients and their PAM50 classification were obtained from TCGA BRCA data available in UCSC Xena. Only BCa samples were included in the present analysis. Using the Hmisc R package, we generated a correlation matrix for each miRNA, applying the Spearman correlation coefficient.

\subsection{Kaplan-Meier Plots}

Overall Survival (OS) was performed for the selected miRNAs using data from miRpower bioinformatic tool (https:/ / kmplot.com, accessed on 3 September 2021) [56]. OS and Relapse Free Survival (RFS) for the selected genes were performed using data from the Kaplan-Meier plotter bioinformatic tool (https:// kmplot.com, accessed on 3 September 2021) [57]. Data was analyzed and plotted using the libraries survminer and survival in R software.

\subsection{Normal, Tumor and Metastatic Gene Expression}

Data and plots comparing normal, tumor and metastatic gene expression were performed using TNMplot bioinformatic tool (https://TNMplot.com, accessed on 1 September 2021) [58], which contains data from 242 normal tissues, 7569 BCa tissues and 82 metastatic tissues. For comparisons among tissues, Kruskal-Wallis followed by Dunn's Test was performed.

Supplementary Materials: The following are available online at https:/ /www.mdpi.com/article/ 10.3390/ijms222011135/s1, Table S1: List of target-genes used to perform Venn's Diagram; Table S2: Target genes and their pathways for hsa-miR-205-5p and miR-143-3p; Table S3: Target genes and their pathways for hsa-miR-21-5p and miR-106b-5p.

Author Contributions: Conceptualization, P.L.F., C.M., R.B.D. and A.D.S.; methodology, P.L.F., C.M., R.B.D. and A.D.S.; software, P.L.F. and E.L.; validation, P.L.F.; formal analysis, P.L.F., C.M. and R.B.D.; investigation, P.L.F., R.B.D., C.M., G.N.D. and K.D.G.; resources, A.D.S.; data curation, P.L.F.; writingoriginal draft preparation, P.L.F. and A.D.S.; writing-review and editing, P.L.F., R.B.D., E.L., G.N.D., K.G. and A.D.S.; visualization, P.L.F.; supervision, A.D.S.; project administration, A.D.S.; funding acquisition, A.D.S. All authors have read and agreed to the published version of the manuscript.

Funding: This research was supported by the Argentinean Agency of Science and Technology (ANPCyT PICT 2014-324; PICT 2015-1345, PICT 2018-1304, PICT START UP-2019-21), Williams Foundation (Argentina), National Cancer Institute (Argentina) (INC 2020) and NIH/NCI Cancer Center Support Grant P30CA013696 (Kevin Gardner), R01 1R0- 1CA253368 (Kevin Gardner). This work was part of Ph.D. thesis of Paula Lucía Farré supported by the CONICET fellowship and National Cancer Institute (Argentina) fellowship. 
Institutional Review Board Statement: The study was approved by the Institutional Ethics Committee of Instituto de Biología y Medicina Experimental (IBYME-CONICET) (protocol code 013/2017, date of approval: 22 May 2017).

Informed Consent Statement: Not applicable, based in public database.

Data Availability Statement: Publicly available datasets were analyzed in this study. This data can be found here: https:/ / xena.ucsc.edu/, accessed on 15 August 2021; http:/ / tripod.nih.gov/ bioplanet/, accessed on 1 August 2021; https:/ /www.genome.jp/kegg/pathway.html, accessed on 1 August 2021; https: / / kmplot.com, accessed on 3 September 2021; https:/ / TNMplot.com, accessed on 1 September 2021.

Acknowledgments: The authors thank Fundación Williams (Argentina) for their support.

Conflicts of Interest: The authors declare no conflict of interest.

\section{References}

1. Bray, F.; Ferlay, J.; Soerjomataram, I.; Siegel, R.L.; Torre, L.A.; Jemal, A. Global cancer statistics 2018: GLOBOCAN estimates of incidence and mortality worldwide for 36 cancers in 185 countries. CA Cancer J. Clin. 2018, 68, 394-424. [CrossRef] [PubMed]

2. Trayes, K.P.; Cokenakes, S.E.H. Breast Cancer Treatment. Am. Fam. Physician 2021, 104, 171-178. [PubMed]

3. Hortobagyi, G.N.; Edge, S.B.; Giuliano, A. New and Important Changes in the TNM Staging System for Breast Cancer. Am. Soc. Clin. Oncol. Educ. Book 2018, 457-467. [CrossRef] [PubMed]

4. Yanovich, G.; Agmon, H.; Harel, M.; Sonnenblick, A.; Peretz, T.; Geiger, T. Clinical proteomics of breast cancer reveals a novel layer of breast cancer classification. Cancer Res. 2018, 78, 6001-6010. [CrossRef]

5. Yin, L.; Duan, J.J.; Bian, X.W.; Yu, S.C. Triple-negative breast cancer molecular subtyping and treatment progress. Breast Cancer Res. 2020, 22, 1-13. [CrossRef]

6. Ha, M.; Kim, V.N. Regulation of microRNA biogenesis. Nat. Rev. Mol. Cell Biol. 2014, 15, 509-524. [CrossRef]

7. Fridrichova, I.; Zmetakova, I. MicroRNAs Contribute to Breast Cancer Invasiveness. Cells 2019, 8, 1361. [CrossRef]

8. Loh, H.-Y.; Norman, B.P.; Lai, K.-S.; Rahman, N.M.A.N.A.; Alitheen, N.B.M.; Osman, M.A. The Regulatory Role of MicroRNAs in Breast Cancer. Int. J. Mol. Sci. 2019, 20, 4940. [CrossRef]

9. Ruan, K.; Fang, X.; Ouyang, G. MicroRNAs: Novel regulators in the hallmarks of human cancer. Cancer Lett. 2009, 285, 116-126. [CrossRef]

10. Bertoli, G.; Cava, C.; Castiglioni, I. Micrornas: New biomarkers for diagnosis, prognosis, therapy prediction and therapeutic tools for breast cancer. Theranostics 2015, 5, 1122-1143. [CrossRef]

11. Kim, S.Y.; Kim, A.Y.; Lee, H.W.; Son, Y.H.; Lee, G.Y.; Lee, J.-W.; Lee, Y.S.; Kim, J.B. miR-27a is a negative regulator of adipocyte differentiation via suppressing PPAR $\gamma$ expression. Biochem. Biophys. Res. Commun. 2010, 392, 323-328. [CrossRef]

12. Alarmo, E.L.; Havunen, R.; Häyrynen, S.; Penkki, S.; Ketolainen, J.; Nykter, M.; Kallioniemi, A. Bone morphogenetic protein 4 regulates microRNA expression in breast cancer cell lines in diverse fashion. Genes Chromosom. Cancer 2016, 55, 227-236. [CrossRef]

13. Kim, Y.C.; Cutler, M.L. Microrna-dependent targeting of rsu1 and the ipp adhesion complex regulates the PTEN/PI3K/AKT signaling pathway in breast cancer cell lines. Int. J. Mol. Sci. 2020, 21, 5458. [CrossRef]

14. Nurzadeh, M.; Naemi, M.; Sheikh Hasani, S. A comprehensive review on oncogenic miRNAs in breast cancer. J. Genet. 2021, 100. [CrossRef]

15. Hong, H.C.; Chuang, C.H.; Huang, W.C.; Weng, S.L.; Chen, C.H.; Chang, K.H.; Liao, K.W.; Huang, H. Da A panel of eight microRNAs is a good predictive parameter for triple-negative breast cancer relapse. Theranostics 2020, 10, 8771-8789. [CrossRef]

16. Goldman, M.J.; Craft, B.; Hastie, M.; Repečka, K.; McDade, F.; Kamath, A.; Banerjee, A.; Luo, Y.; Rogers, D.; Brooks, A.N.; et al. Visualizing and interpreting cancer genomics data via the Xena platform. Nat. Biotechnol. 2020, 38, 675-678. [CrossRef]

17. Liu, M.; Zhou, S.; Wang, J.; Zhang, Q.; Yang, S.; Feng, J.; Xu, B.; Zhong, S. Identification of genes associated with survival of breast cancer patients. Breast Cancer 2019, 26, 317-325. [CrossRef]

18. Chang, J.T.-H.; Wang, F.; Chapin, W.; Huang, R.S. Identification of MicroRNAs as breast cancer prognosis markers through the cancer genome atlas. PLoS ONE 2016, 11, e0168284. [CrossRef]

19. Lu, J.; Tan, T.; Zhu, L.; Dong, H.; Xian, R. Hypomethylation Causes MIR21 Overexpression in Tumors. Mol. Ther.-Oncolytics 2020, 18, 47-57. [CrossRef]

20. Qian, B.; Katsaros, D.; Lu, L.; Preti, M.; Durando, A.; Arisio, R.; Mu, L.; Yu, H. High miR-21 expression in breast cancer associated with poor disease-free survival in early stage disease and high TGF- $\beta 1$. Breast Cancer Res. Treat. 2009, 117, 131-140. [CrossRef]

21. Haakensen, V.D.; Nygaard, V.; Greger, L.; Aure, M.R.; Fromm, B.; Bukholm, I.R.K.; Lüders, T.; Chin, S.F.; Git, A.; Caldas, C.; et al. Subtype-specific micro-RNA expression signatures in breast cancer progression. Int. J. Cancer 2016, 139, 1117-1128. [CrossRef]

22. Yu, X.; Liang, J.; Xu, J.; Li, X.; Xing, S.; Li, H.; Liu, W.; Liu, D.; Xu, J.; Huang, L.; et al. Identification and validation of circulating microRNA signatures for breascancer early detection based on large scale tissue-derived data. J. Breast Cancer 2018, 21, 363-370. [CrossRef] 
23. Pourteimoor, V.; Paryan, M.; Mohammadi-Yeganeh, S. microRNA as a systemic intervention in the specific breast cancer subtypes with C-MYC impacts; introducing subtype-based appraisal tool. J. Cell. Physiol. 2018, 233, 5655-5669. [CrossRef]

24. Mango, V.L.; Al-Khawari, H.; Dershaw, D.D.; Ashkanani, M.H.; Pennisi, B.; Turner, P.; Thornton, C.; Morris, E.A. Initiating a National Mammographic Screening Program: The Kuwait Experience Training with a US Cancer Center. J. Am. Coll. Radiol. 2019, 16, 202-207. [CrossRef]

25. Wang, H.; Tan, Z.; Hu, H.; Liu, H.; Wu, T.; Zheng, C.; Wang, X.; Luo, Z.; Wang, J.; Liu, S.; et al. MicroRNA-21 promotes breast cancer proliferation and metastasis by targeting LZTFL1. BMC Cancer 2019, 19. [CrossRef]

26. Newie, I.; Søkilde, R.; Persson, H.; Jacomasso, T.; Gorbatenko, A.; Borg, Å.; De Hoon, M.; Pedersen, S.F.; Rovira, C. HER2-encoded mir-4728 forms a receptor-independent circuit with miR-21-5p through the non-canonical poly(A) polymerase PAPD5. Sci. Rep. 2016, 6. [CrossRef]

27. Calvano Filho, C.M.C.; Calvano-Mendes, D.C.; Carvalho, K.C.; Maciel, G.A.; Ricci, M.D.; Torres, A.P.; Filassi, J.R.; Baracat, E.C. Triple-negative and luminal A breast tumors: Differential expression of miR-18a-5p, miR-17-5p, and miR-20a-5p. Tumor Biol. 2014, 35, 7733-7741. [CrossRef]

28. Moi, L.; Braaten, T.; Al-Shibli, K.; Lund, E.; Busund, L.T.R. Differential expression of the miR-17-92 cluster and miR-17 family in breast cancer according to tumor type; Results from the Norwegian Women and Cancer (NOWAC) study. J. Transl. Med. 2019, 17, 1-20. [CrossRef]

29. De Rinaldis, E.; Gazinska, P.; Mera, A.; Modrusan, Z.; Fedorowicz, G.M.; Burford, B.; Gillett, C.; Marra, P.; Grigoriadis, A.; Dornan, D.; et al. Integrated genomic analysis of triple-negative breast cancers reveals novel microRNAs associated with clinical and molecular phenotypes and sheds light on the pathways they control. BMC Genom. 2013, 14, 1. [CrossRef]

30. Zheng, R.; Pan, L.; Gao, J.; Ye, X.; Chen, L.; Zhang, X.; Tang, W.; Zheng, W. Prognostic value of miR-106b expression in breast cancer patients. J. Surg. Res. 2015, 195, 158-165. [CrossRef]

31. Guarnieri, A.L.; Towers, C.G.; Drasin, D.J.; Oliphant, M.U.J.; Andrysik, Z.; Hotz, T.J.; Vartuli, R.L.; Linklater, E.S.; Pandey, A.; Khanal, S.; et al. The miR-106b-25 cluster mediates breast tumor initiation through activation of NOTCH1 via direct repression of NEDD4L. Oncogene 2018, 37, 3879-3893. [CrossRef] [PubMed]

32. Tan, W.; Liang, G.; Xie, X.; Jiang, W.; Tan, L.; Sanders, A.J.; Liu, Z.; Ling, Y.; Zhong, W.; Tian, Z.; et al. Incorporating MicroRNA into Molecular Phenotypes of Circulating Tumor Cells Enhances the Prognostic Accuracy for Patients with Metastatic Breast Cancer. Oncologist 2019, 24. [CrossRef] [PubMed]

33. Chen, K.-H.E.; Bustamante, K.; Nguyen, V.; Walker, A.M. Involvement of miR-106b in tumorigenic actions of both prolactin and estradiol. Oncotarget 2017, 8, 36368-36382. [CrossRef] [PubMed]

34. Wang, Z.; Li, T.E.; Chen, M.; Pan, J.J.; Shen, K.W. miR-106b-5p contributes to the lung metastasis of breast cancer via targeting CNN1 and regulating Rho/ROCK1 pathway. Aging 2020, 12, 1867-1887. [CrossRef] [PubMed]

35. Gong, C.; Qu, S.; Liu, B.; Pan, S.; Jiao, Y.; Nie, Y.; Su, F.; Liu, Q.; Song, E. MiR-106b expression determines the proliferation paradox of TGF- $\beta$ in breast cancer cells. Oncogene 2015, 34, 84-93. [CrossRef] [PubMed]

36. Schrijver, W.A.M.E.; van Diest, P.J.; Moelans, C.B. Unravelling site-specific breast cancer metastasis: A microRNA expression profiling study. Oncotarget 2017, 8, 3111-3123. [CrossRef] [PubMed]

37. Lee, C.M.; Chung, I.Y.; Park, Y.; Yun, K.W.; Jo, H.G.; Park, H.J.; Lee, H.J.; Lee, S.B.; Kim, H.J.; Ko, B.S.; et al. The impact of androgen receptor and histone deacetylase 1 expression on the prognosis of ductal carcinoma in situ. J. Breast Cancer 2020, 23, 610-621. [CrossRef]

38. Sanli, T.; Linher-Melville, K.; Tsakiridis, T.; Singh, G. Sestrin2 modulates AMPK subunit expression and its response to ionizing radiation in breast cancer cells. PLoS ONE 2012, 7. [CrossRef]

39. Cordani, M.; Butera, G.; Dando, I.; Torrens-Mas, M.; Butturini, E.; Pacchiana, R.; Oppici, E.; Cavallini, C.; Gasperini, S.; Tamassia, N.; et al. Mutant p53 blocks SESN1/AMPK/PGC-1 $\alpha / \mathrm{UCP} 2$ axis increasing mitochondrial $\mathrm{O}_{2}{ }^{-\cdot}$ production in cancer cells. Br. J. Cancer 2018, 119, 994-1008. [CrossRef]

40. Cordani, M.; Oppici, E.; Dando, I.; Butturini, E.; Dalla Pozza, E.; Nadal-Serrano, M.; Oliver, J.; Roca, P.; Mariotto, S.; Cellini, B.; et al. Mutant p53 proteins counteract autophagic mechanism sensitizing cancer cells to mTOR inhibition. Mol. Oncol. 2016, 10, 1008-1029. [CrossRef]

41. Veeraraghavan, J.; Tan, Y.; Cao, X.X.; Kim, J.A.; Wang, X.; Chamness, G.C.; Maiti, S.N.; Cooper, L.J.N.; Edwards, D.P.; Contreras, A.; et al. Recurrent ESR1-CCDC170 rearrangements in an aggressive subset of oestrogen receptor-positive breast cancers. Nat. Commun. 2014, 5. [CrossRef]

42. Ortiz-Padilla, C.; Gallego-Ortega, D.; Browne, B.C.; Hochgräfe, F.; Caldon, C.E.; Lyons, R.J.; Croucher, D.R.; Rickwood, D.; Ormandy, C.J.; Brummer, T.; et al. Functional characterization of cancer-associated Gab1 mutations. Oncogene 2013, 32, $2696-2702$. [CrossRef]

43. Mouradian, M.; Kikawa, K.D.; Johnson, E.D.; Beck, K.L.; Pardini, R.S. Key roles for GRB2-associated-binding protein 1, phosphatidylinositol-3-kinase, cyclooxygenase 2, prostaglandin E2 and transforming growth factor alpha in linoleic acid-induced upregulation of lung and breast cancer cell growth. Prostaglandins Leukot. Essent. Fat. Acids 2014, 90, 105-115. [CrossRef]

44. Gensler, M.; Buschbeck, M.; Ullrich, A. Negative Regulation of HER2 Signaling by the PEST-type Protein-tyrosine Phosphatase BDP1. J. Biol. Chem. 2004, 279, 12110-12116. [CrossRef]

45. Wang, X.; Peng, J.; Yang, Z.; Zhou, P.J.; An, N.; Wei, L.; Zhu, H.H.; Lu, J.; Fang, Y.X.; Gao, W.Q. Elevated expression of Gab1 promotes breast cancer metastasis by dissociating the PAR complex. J. Exp. Clin. Cancer Res. 2019, 38. [CrossRef] 
46. Escamilla-Powers, J.R.; Daniel, C.J.; Farrell, A.; Taylor, K.; Zhang, X.; Byers, S.; Sears, R. The tumor suppressor protein HBP1 is a novel c-Myc-binding protein that negatively regulates c-Myc transcriptional activity. J. Biol. Chem. 2010, 285, 4847-4858. [CrossRef]

47. Paulson, K.E.; Rieger-Christ, K.; McDevitt, M.A.; Kuperwasser, C.; Kim, J.; Unanue, V.E.; Zhang, X.; Hu, M.; Ruthazer, R.; Berasi, S.P.; et al. Alterations of the HBP1 transcriptional repressor are associated with invasive breast cancer. Cancer Res. 2007, 67, 6136-6145. [CrossRef]

48. Kim, J.; Zhang, X.; Rieger-Christ, K.M.; Summerhayes, I.C.; Wazer, D.E.; Paulson, K.E.; Yee, A.S. Suppression of Wnt signaling by the green tea compound (-)-epigallocatechin 3-gallate (EGCG) in invasive breast cancer cells: Requirement of the transcriptional repressor HBP1. J. Biol. Chem. 2006, 281, 10865-10875. [CrossRef]

49. Li, H.; Bian, C.; Liao, L.; Li, J.; Zhao, R.C. miR-17-5p promotes human breast cancer cell migration and invasion through suppression of HBP1. Breast Cancer Res. Treat. 2011, 126, 565-575. [CrossRef]

50. Bollaert, E.; de Rocca Serra, A.; Demoulin, J.B. The HMG box transcription factor HBP1: A cell cycle inhibitor at the crossroads of cancer signaling pathways. Cell. Mol. Life Sci. 2019, 76, 1529-1539. [CrossRef]

51. Farré, P.L.; Scalise, G.D.; Duca, R.B.; Dalton, G.N.; Massillo, C.; Porretti, J.; Graña, K.; Gardner, K.; De Luca, P.; De Siervi, A. CTBP1 and metabolic syndrome induce an mRNA and miRNA expression profile critical for breast cancer progression and metastasis. Oncotarget 2018, 9, 13848-13858. [CrossRef] [PubMed]

52. Dalton, G.N.; Massillo, C.; Scalise, G.D.; Duca, R.; Porretti, J.; Farré, P.L.; Gardner, K.; Paez, A.; Gueron, G.; De Luca, P.; et al. CTBP1 depletion on prostate tumors deregulates miRNA/mRNA expression and impairs cancer progression in metabolic syndrome mice. Cell Death Dis. 2019, 10, 299. [CrossRef]

53. Porretti, J.; Dalton, G.N.; Massillo, C.; Scalise, G.D.; Farré, P.L.; Elble, R.; Gerez, E.N.; Accialini, P.; Cabanillas, A.M.; Gardner, K.; et al. CLCA2 epigenetic regulation by CTBP1, HDACs, ZEB1, EP300 and miR-196b-5p impacts prostate cancer cell adhesion and EMT in metabolic syndrome disease. Int. J. Cancer 2018, 143, 897-906. [CrossRef]

54. Chen, C.; Ridzon, D.A.; Broomer, A.J.; Zhou, Z.; Lee, D.H.; Nguyen, J.T.; Barbisin, M.; Xu, N.L.; Mahuvakar, V.R.; Andersen, M.R.; et al. Real-time quantification of microRNAs by stem-loop RT-PCR. Nucleic Acids Res. 2005, 33, e179. [CrossRef]

55. Karagkouni, D.; Paraskevopoulou, M.D.; Chatzopoulos, S.; Vlachos, I.S.; Tastsoglou, S.; Kanellos, I.; Papadimitriou, D.; Kavakiotis, I.; Maniou, S.; Skoufos, G.; et al. DIANA-TarBase v8: A decade-long collection of experimentally supported miRNA-gene interactions. Nucleic Acids Res. 2018, 46, D239-D245. [CrossRef]

56. Lánczky, A.; Nagy, Á.; Bottai, G.; Munkácsy, G.; Szabó, A.; Santarpia, L.; Győrffy, B. miRpower: A web-tool to validate survivalassociated miRNAs utilizing expression data from 2178 breast cancer patients. Breast Cancer Res. Treat. 2016, 160, 439-446. [CrossRef]

57. Győrffy, B. Survival analysis across the entire transcriptome identifies biomarkers with the highest prognostic power in breast cancer. Comput. Struct. Biotechnol. J. 2021, 19, 4101-4109. [CrossRef]

58. Bartha, Á.; Győrffy, B. TNMplot.com: A Web Tool for the Comparison of Gene Expression in Normal, Tumor and Metastatic Tissues. Int. J. Mol. Sci. 2021, 22, 2622. [CrossRef] 